\title{
Higher Approximations to Study Statistical Characteristics of Waves in Multiscale Inhomogeneous Media
}

\begin{abstract}
M. V. Tinin (iD
Irkutsk State University, 20 Gagarin Blvd, Irkutsk 664003, Russia

Correspondence should be addressed to M. V. Tinin; mtinin@api.isu.ru

Received 29 April 2017; Accepted 4 January 2018; Published 7 February 2018

Academic Editor: Xavier Leoncini

Copyright ( 2018 M. V. Tinin. This is an open access article distributed under the Creative Commons Attribution License, which permits unrestricted use, distribution, and reproduction in any medium, provided the original work is properly cited.

The previously obtained integral field representation in the form of double weighted Fourier transform (DWFT) describes effects of inhomogeneities with different scales. The first DWFT approximation describing the first-order effects does not account for incident wave distortions. However, in inhomogeneous media the multiscale second-order effects can also take place when largescale inhomogeneities distort the field structure of the wave incident on small-scale inhomogeneities. The paper presents the results of the use of DWFT to derive formulas for wave statistical moments with respect to the first- and second-order effects. It is shown that, for narrow-band signals, the second-order effects do not have a significant influence on the frequency correlation. We can neglect the contribution of the second-order effects to the spatial intensity correlation when thickness of the inhomogeneous layer is small, but these effects become noticeable as the layer thickness increases. Accounting for the second-order effects enabled us to get a spatial intensity correlation function, which at large distances goes to the results obtained earlier by the path integral method. This proves that the incident wave distortion effects act on the intensity fluctuations of a wave propagating in a multiscale randomly inhomogeneous medium.
\end{abstract}

\section{Introduction}

The presence of random inhomogeneities in a radio signal path can have a profound effect on functioning of radiotechnical systems. Fluctuations (scintillations) in a signal in the randomly inhomogeneous ionosphere, in particular, reduce resolving power of the global Earth satellite monitoring and the rate of data transfer in communication systems. On the other hand, observations of scintillation of radio signals from such extraterrestrial sources as stars and artificial Earth satellites contribute significantly to our knowledge of the inhomogeneous structure of the ionosphere and troposphere.

The methods for describing scintillation developed in the $60-80$ s of the last century used the geometrical optics (GO) and Born and Rytov approximations [1-9], as well as the phase-screen method [2, 3, 7, 10-12]. The GO approximation model can only be adopted to describe the effects of inhomogeneities with transverse scales exceeding the Fresnel radius. The effects of inhomogeneities with scales smaller than the Fresnel radius (Fresnel diffraction effects) cannot be correctly described within the GO model.
It is possible to take account of the Fresnel diffraction effects using the perturbations theory. The Born approximation (single scattering theory) uses the perturbation theory to describe the field itself, and in the Rytov approximation, the perturbation theory is employed to derive the complex phase associated with the field logarithm. However, the Rytov and Born approximations take the diffraction Fresnel effects into account only for weak fluctuations; these approximations are not applicable in multipath emergence and in strong focusing on random caustics. Most of the approaches to simulation of the latter effects are based on integral field representations. When the inhomogeneities are in a restricted area away from the source and the observer and the intensity of inhomogeneities is rather weak, it is possible to use GO $[10,11]$ or Rytov [12] approximations near the inhomogeneous area. To get the field integral representation in this situation, accounting for the diffraction effects and caustics arising away from inhomogeneities, often the Kirchhoff method $[2,5-7,13]$ is applied, which goes to the phase-screen method when the inhomogeneous layer thickness is reduced $[2,3,7]$. 
This approach is extended by the path integral method $[14,15]$ and the multiple phase-screen method $[11,16,17]$. However, the path integral method represents a solution in the form of a two-dimensional integral continuum. And the second method involves numerical integration, finite but still large enough (up to 20 for optical radiation in turbulent atmosphere [18]) number of phase screens, which makes it difficult to use these methods for diagnostic purposes.

In the Markov approximation, equations for statistical moments [2] are obtained from the wave equation in the small-angle approximation. Unfortunately, using analytics, we can find solutions only to equations for the mean field and for the spatial correlation function [2]. Equations for other moments can be solved with either numerical [19] or asymptotic methods [20].

Another approach involves the Maslov and interference integral methods [21, 22] and relies on the expanding of the solution of the wave equation in plane waves. This approach requires knowledge of a ray field structure. In a number of problems such as wave propagation in random media and when diagnosing inhomogeneous media, this structure is not always known.

Combinations of the Maslov and interference integral methods were used in solving equations for the two-point random function (averaging of which gives the coherence function) [23] and Markov equations for statistical moments [24]. The same approach enabled solving the equation for the product of the desired field and the known solution (termed as "joker wave" in [25]) in extended 3D space for 2D problem [26] and in the extended 5D space for 3D problem [26-29]. The resulting integral representation in the form of a double weighted Fourier transform (DWFT) describes the wave field, considering for simultaneous effects of inhomogeneities of different scales [26-29].

We will use here the DWFT method [26-30], which in the small-angle approximation describes both strong fluctuations associated with the random caustics that form in a large-scale inhomogeneous medium, and the Fresnel type diffraction effects typical for wave propagation in a medium with smallscale inhomogeneities. Unlike the Maslov and interference integral methods, where the Fourier transform is performed in the receiver or transmitter coordinates, respectively, the DWFT method uses the Fourier transform with respect to both receiver and transmitter coordinates. As a result, in the DWFT integral representation, the wave field is expanded in partial waves that do not have amplitude fluctuations in the first approximation. This not only makes it easier to find the statistical moments of the field, but also shows a greater validity range of the DWFT method in statistical problems compared to the Maslov and interference integral methods.

Let us remark that, in the DWFT method, as in other GO generalizations to generate a wave solution, we use "spanning a wave-field cloth on a ray framework" [21]. In the first DWFT approximation [26-30], the contribution of lowamplitude inhomogeneities to the partial wave phase takes the integral from permittivity perturbation along the unperturbed (direct) ray. For such an effect that inhomogeneities of different scales impose on the wave field we shall call the firstorder effect. In addition, in a multiscale medium, large-scale inhomogeneities distort the field structure of a wave incident on a small-scale inhomogeneity. At the same time, keeping in mind the several order difference of scales and amplitudes of various inhomogeneities, the distortion of an incident wave on the scales of small-scale inhomogeneities can be significant. The effects which require trajectory variations to be taken into account when it passes through an inhomogeneity will be called the second-order effects.

Here we use the DWFT method when analyzing statistical characteristics of a wave propagating in a multiscale randomly inhomogeneous medium, with and without regard to the second-order effects. The next, second, section describes the DWFT method. The third section employs the perturbation theory in DWFT and shows transition of the second DWFT approximation to the second GO approximation. The fourth section focuses on the use of the first DWFT approximation to derive expressions for the mean field and the twofrequency mutual coherence function. In the fifth section, the second DWFT approximation considers the secondorder effects. Also, the expression is derived for the intensity correlation function, and we compare it asymptotically over long distances, with regard to the second-order effects, with the known results of the path integral method. The conclusion summarizes the main results.

\section{Method of Double Weighted Fourier Transform (DWFT)}

Let us consider the field of a harmonic (with time dependence of the form $\exp (-i \omega t))$ point source located at the point $\mathbf{r}_{0}=$ $\left\{x_{0}, y_{0}, z_{0}\right\}=\left\{\boldsymbol{\rho}_{0}, z_{0}\right\}$. In a scalar approximation, this field in an inhomogeneous medium is a solution of equation

$$
\Delta E\left(\mathbf{r}, \mathbf{r}_{0}\right)+k^{2} \varepsilon(\mathbf{r}) E\left(\mathbf{r}, \mathbf{r}_{0}\right)=A_{0} \delta\left(\mathbf{r}-\mathbf{r}_{0}\right),
$$

where permittivity $\varepsilon(\mathbf{r})=\bar{\varepsilon}(\mathbf{r})+\widetilde{\varepsilon}(\mathbf{r})$ includes the background (deterministic) $\bar{\varepsilon}(\mathbf{r})$ and random $\widetilde{\varepsilon}(\mathbf{r})$ components, respectively; $A_{0}$ is the amplitude of the incident spherical wave; $k=\omega / c=2 \pi / \lambda$ is the wave number; $c$ and $\lambda$ are the light speed and the wavelength in free space, respectively. Let $\bar{\varepsilon}(\mathbf{r})=1$; that is, we will consider the effects of random inhomogeneities in the absence of a background inhomogeneity. Quite often, the size $l_{\varepsilon}$ of inhomogeneities exceeds the wavelength such that $k l_{\varepsilon} \gg 1$. In this case, a wave is scattered along its propagation path, for example, along the axis $z$, and usually [2-4] by substituting

$$
E\left(\mathbf{r}, \mathbf{r}_{0}\right)=U\left(\mathbf{r}, \mathbf{r}_{0}\right) \exp \left[i k\left(z-z_{0}\right)\right]
$$

and from elliptical equation (1) we pass on to the parabolic equation for the "complex amplitude" $U\left(\mathbf{r}, \mathbf{r}_{0}\right)$ :

$$
2 i k \frac{\partial}{\partial z} U(\boldsymbol{\rho}, z)+\frac{\partial^{2}}{\partial \rho^{2}} U(\boldsymbol{\rho}, z)+k^{2} \widetilde{\mathcal{\varepsilon}}(\mathbf{r}) U(\boldsymbol{\rho}, z)=0
$$

with the initial condition

$$
U\left(\boldsymbol{\rho}, z_{0}\right)=\frac{-i A_{0} \delta\left(\mathbf{r}-\mathbf{r}_{0}\right)}{(2 k)} .
$$


In the absence of inhomogeneity with $\widetilde{\mathcal{\varepsilon}}(\mathbf{r})=0$ and $A_{0}=1$, the solution of problem (3)-(4) takes the form of

$$
\begin{aligned}
& U(\boldsymbol{\rho}, z)=U_{0}(\boldsymbol{\rho}, z) \\
& \quad=-A_{0}\left(4 \pi\left(z-z_{0}\right)\right)^{-1} \exp \left\{\frac{i k 0.5\left(\rho-\rho_{0}\right)^{2}}{\left(z-z_{0}\right)}\right\} .
\end{aligned}
$$

Let us introduce a five-variable function as the product of the unknown function $U\left(\rho_{1}, z\right)$ and the known function $U_{2}^{*}\left(\rho_{2}, z\right)$.

$$
\mathrm{V}\left(\boldsymbol{\rho}_{1}, \boldsymbol{\rho}_{2}, z\right)=U\left(\boldsymbol{\rho}_{1}, z\right) U_{2}^{*}\left(\boldsymbol{\rho}_{2}, z\right)
$$

For function $U_{2}{ }^{*}\left(\boldsymbol{\rho}_{2}, z\right)$, sometimes called a "joker wave" [25], we will take $U_{0}{ }^{*}\left(\rho_{2}, z\right)$, which satisfies the equation

$$
\begin{aligned}
\frac{2 i k \partial U_{2}{ }^{*}}{\partial z}-\frac{\partial^{2} U_{2}{ }^{*}}{\partial \rho_{2}^{2}} & =0 \\
U_{2}{ }^{*}\left(\boldsymbol{\rho}_{a}, z_{0}\right) & =\frac{i \delta\left(\boldsymbol{\rho}_{2}-\boldsymbol{\rho}_{20}\right)}{(2 k)} .
\end{aligned}
$$

Taking into account (3)-(4) and (7), we can write an equation for $\mathrm{V}\left(\boldsymbol{\rho}_{1}, \boldsymbol{\rho}_{2}, z\right)$ :

$$
\begin{aligned}
& \frac{\partial \mathrm{V}}{\partial z}-\frac{i \partial^{2} \mathrm{~V}}{\left(k \partial \boldsymbol{\rho}_{+} \partial \boldsymbol{\rho}_{-}\right)}-i 0.5 k \widetilde{\varepsilon}\left(\boldsymbol{\rho}_{+}+\frac{\boldsymbol{\rho}_{-}}{2}, z\right) \mathrm{V}=0, \\
& \mathrm{~V}\left(\boldsymbol{\rho}_{+}, \boldsymbol{\rho}_{-}, z_{0}\right)=\frac{A_{0} \delta\left(\boldsymbol{\rho}_{+}-\boldsymbol{\rho}_{0+}\right) \delta\left(\boldsymbol{\rho}_{-}-\boldsymbol{\rho}_{0-}\right)}{(2 k)^{2}}
\end{aligned}
$$

In (8), we introduce new variables:

$$
\begin{gathered}
\boldsymbol{\rho}_{+}=\frac{\left(\boldsymbol{\rho}_{1}+\boldsymbol{\rho}_{2}\right)}{2}, \\
\boldsymbol{\rho}_{-}=\boldsymbol{\rho}_{1}-\boldsymbol{\rho}_{2}, \\
\boldsymbol{\rho}_{0+}=\frac{\left(\boldsymbol{\rho}_{01}+\boldsymbol{\rho}_{02}\right)}{2}, \\
\boldsymbol{\rho}_{0-}=\boldsymbol{\rho}_{01}-\boldsymbol{\rho}_{02} .
\end{gathered}
$$

Let us turn from the function $\mathrm{V}\left(\boldsymbol{\rho}_{1}, \boldsymbol{\rho}_{2}, z\right)$ to its Fourier image with respect to variables $\boldsymbol{\rho}_{0+}$ and $\boldsymbol{\rho}_{-}$:

$$
\begin{aligned}
& \mathrm{V}\left(\boldsymbol{\rho}_{+}, \boldsymbol{\rho}_{-}, z\right)=\int_{-\infty}^{\infty} \int_{-\infty}^{\infty} \int_{-\infty}^{\infty} \int_{-\infty}^{\infty} d^{2} s d^{2} p \widehat{\mathrm{V}}(s, p, z) \\
& \cdot \exp \left[-i k\left(2 \boldsymbol{\rho}_{0+} \mathbf{s}-\mathbf{p} \boldsymbol{\rho}_{-}\right)\right] \\
& \widehat{\mathrm{V}}(s, p, z)=0.25\left(\frac{k}{\pi}\right)^{4} \\
& \quad \cdot \int_{-\infty}^{\infty} \int_{-\infty}^{\infty} \int_{-\infty}^{\infty} \int_{-\infty}^{\infty} d^{2} \rho_{0+} d^{2} \rho_{-} \mathrm{V}\left(\boldsymbol{\rho}_{+}, \boldsymbol{\rho}_{-}, z\right) \\
& \cdot \exp \left[i k\left(2 \boldsymbol{\rho}_{0+} \mathbf{s}-\mathbf{p} \boldsymbol{\rho}_{-}\right)\right] .
\end{aligned}
$$

From (8), we get the equation for $\widehat{\mathrm{V}}(s, p, z)$ :

$$
\begin{aligned}
& \frac{\partial \widehat{V}}{\partial z}+\frac{\mathbf{p} \partial \widehat{V}}{\partial \boldsymbol{\rho}_{+}}-i 0.5 k \widetilde{\varepsilon}\left(\boldsymbol{\rho}_{+}-\frac{\partial}{(2 i k \partial \mathbf{p})}, z\right) \widehat{\mathrm{V}}=0, \\
& \widehat{\mathrm{V}}\left(\mathbf{s}, \mathbf{p}, z_{0}\right)=\frac{A_{0} k^{2}}{(2 \pi)^{4}} \exp \left[i k\left(2 \boldsymbol{\rho}_{+} \mathbf{s}-\mathbf{p} \boldsymbol{\rho}_{0_{-}}\right)\right] .
\end{aligned}
$$

It is easy to find a solution to (12) in the absence of inhomogeneities, that is, if $\widetilde{\varepsilon}=0$,

$$
\begin{aligned}
\widehat{\mathrm{V}} & =\widehat{\mathrm{V}}_{0} \\
& =\frac{A_{0} k^{2}}{(2 \pi)^{4}} \exp \left\{i k\left[2 \boldsymbol{\rho}_{+} \mathbf{s}-2 \mathbf{s p}\left(z-z_{0}\right)-\mathbf{p} \boldsymbol{\rho}_{0-}\right]\right\} .
\end{aligned}
$$

In the GO method and its generalizations, solution of the wave equation with large $k$ is presented as a Debye series in inverse powers of $k$. Here, we will also find the solution of problem (12) in the form of

$$
\widehat{\mathrm{V}}=\sum_{0}^{\infty} \frac{A^{(m)}}{(i k)^{m}} \exp (i k \varphi) .
$$

Substituting (14) into (12) and equating coefficients of equal power in $k$, we derive a system of recurrent equations for $\varphi$ and $A^{(m)}$. If we limit (14) to the first term, we get

$$
\widehat{\mathrm{V}} \approx A^{(0)} \exp (i k \varphi)=A \exp (i k \varphi)
$$

where the phase path (eikonal) $\varphi$ and the amplitude $A$ satisfy equations

$$
\begin{gathered}
\frac{\partial \varphi}{\partial z}+\mathbf{p} \frac{\partial \varphi}{\partial \boldsymbol{\rho}_{+}}-\frac{1}{2} \widetilde{\mathcal{\varepsilon}}\left(\boldsymbol{\rho}_{+}-\frac{1}{2} \frac{\partial \varphi}{\partial \mathbf{p}}\right)=0, \\
\frac{\partial A}{\partial z}+\mathbf{p} \frac{\partial A}{\partial \boldsymbol{\rho}_{+}}+\frac{A}{8} \frac{\partial^{2}}{\partial \boldsymbol{\rho}_{+} \partial \mathbf{p}} \widetilde{\mathcal{\varepsilon}}\left(\boldsymbol{\rho}_{+}-\frac{1}{2} \frac{\partial \varphi}{\partial \mathbf{p}}\right) \\
+\frac{1}{4} \frac{\partial A}{\partial \mathbf{p}} \frac{\partial}{\partial \boldsymbol{\rho}_{+}} \widetilde{\mathcal{\varepsilon}}\left(\boldsymbol{\rho}_{+}-\frac{1}{2} \frac{\partial \varphi}{\partial \mathbf{p}}\right)=0 .
\end{gathered}
$$

Initial conditions for eikonal (16) and transfer (17) equations can be obtained from (13):

$$
\begin{aligned}
& \left.\varphi\right|_{z=z_{0}}=2 s \boldsymbol{\rho}_{+}-\mathbf{p} \boldsymbol{\rho}_{0-}, \\
& \left.A\right|_{z=z_{0}}=\frac{A_{0} k^{2}}{(2 \pi)^{4}} .
\end{aligned}
$$

Solving equations in partial first-order derivatives (16)-(17) can be reduced to solving equations of characteristics [1], and thus from (2), (6), (10), we obtain the following expression:

$$
\begin{aligned}
E(\boldsymbol{\rho}, z)= & -A_{0} \frac{k^{2}\left(z-z_{0}\right)}{4 \pi^{3}} \\
& \cdot \exp \left\{i k\left[z-z_{0}+\frac{\left(\boldsymbol{\rho}_{2}-\boldsymbol{\rho}_{02}\right)^{2}}{2\left(z-z_{0}\right)}\right]\right\} \\
& \cdot \int_{-\infty}^{\infty} \int_{-\infty}^{\infty} d^{2} s d^{2} p \exp \{-\gamma+i k \varphi(\mathbf{s}, \mathbf{p})\},
\end{aligned}
$$


where

$$
\begin{aligned}
& \varphi(\mathbf{s}, \mathbf{p})=2 \mathbf{s}\left(\boldsymbol{\rho}_{+}+\boldsymbol{\rho}_{+}\left(z_{0}\right)-\boldsymbol{\rho}_{0+}\right)+\mathbf{p}\left(\boldsymbol{\rho}_{-}-2 \boldsymbol{\rho}_{-}(z)\right. \\
& \left.-\boldsymbol{\rho}_{0-}\right)+2 \int_{z 0}^{z} \mathbf{p}_{-}\left(z^{\prime}\right) \mathbf{p}_{+}\left(z^{\prime}\right) d z^{\prime}+0.5 \int_{z 0}^{z} \widetilde{\varepsilon}\left[\boldsymbol{\rho}_{+}\right. \\
& \left.+\frac{\boldsymbol{\rho}_{0-}}{2}+\boldsymbol{\rho}_{+}\left(z^{\prime}\right)+\boldsymbol{\rho}_{-}\left(z^{\prime}\right), z^{\prime}\right] d z^{\prime}, \\
& \gamma=0.125 \int_{z 0}^{z} \frac{\partial^{2}}{\partial \rho_{+}^{2}} \widetilde{\varepsilon}\left[\boldsymbol{\rho}_{+}+\frac{\boldsymbol{\rho}_{0-}}{2}+\boldsymbol{\rho}_{+}\left(z^{\prime}\right)+\boldsymbol{\rho}_{-}\left(z^{\prime}\right),\right. \\
& \left.z^{\prime}\right] \frac{\partial}{\partial p} \boldsymbol{\rho}_{-}\left(z^{\prime}\right) d z^{\prime} .
\end{aligned}
$$

In (20), integration is performed along the characteristics (rays) that satisfy ray equations

$$
\begin{aligned}
& \frac{\partial \mathbf{p}_{+,-}\left(z^{\prime}\right)}{\partial z^{\prime}} \\
& \quad=\frac{1}{4} \frac{\partial}{\partial \boldsymbol{\rho}_{+}} \widetilde{\varepsilon}\left[\boldsymbol{\rho}_{+}+\frac{\boldsymbol{\rho}_{0-}}{2}+\boldsymbol{\rho}_{+}\left(z^{\prime}\right)+\boldsymbol{\rho}_{-}\left(z^{\prime}\right), z^{\prime}\right], \\
& \frac{\partial \boldsymbol{\rho}_{+,-}\left(z^{\prime}\right)}{\partial z^{\prime}}=\mathbf{p}_{+,-}\left(z^{\prime}\right),
\end{aligned}
$$

with initial conditions

$$
\begin{aligned}
\boldsymbol{\rho}_{+}(z) & =\boldsymbol{\rho}_{-}\left(z_{0}\right)=0, \\
\mathbf{p}_{-}\left(z_{0}\right) & =\mathbf{s}, \\
\mathbf{p}_{+}(z) & =\mathbf{p} .
\end{aligned}
$$

Our solution (19) contains "excess" variables $\boldsymbol{\rho}_{02}$ and $\boldsymbol{\rho}_{2}$. They can be expressed through coordinates $\boldsymbol{\rho}_{01}$ and $\boldsymbol{\rho}_{1}$. This corresponds to the transition to a certain surface in the fivedimensional space $\left\{\boldsymbol{\rho}_{1}, \boldsymbol{\rho}_{2}, z\right\}$. In particular, just like in [26], we can choose this connection from the condition that there are no transverse coordinates $\boldsymbol{\rho}_{+}, \boldsymbol{\rho}_{0-}$ in the arguments of the function $\widetilde{\varepsilon}(\rho, z)$ :

$$
\boldsymbol{\rho}_{+}+\frac{\boldsymbol{\rho}_{0-}}{2}=\frac{\left(\boldsymbol{\rho}_{1}+\boldsymbol{\rho}_{2}+\boldsymbol{\rho}_{01}-\boldsymbol{\rho}_{02}\right)}{2}=0 .
$$

In this case, having set $\boldsymbol{\rho}_{1}=\boldsymbol{\rho}, \boldsymbol{\rho}_{01}=\boldsymbol{\rho}_{0}$ and taking (5) into account, (19)-(21) can be written as follows:

$$
\begin{aligned}
E(\boldsymbol{\rho}, z)= & E_{0}(\boldsymbol{\rho}, z)\left[\frac{k\left(z-z_{0}\right)}{\pi}\right]^{2} \exp \left\{i k \frac{2 \boldsymbol{\rho} \boldsymbol{\rho}_{0}}{\left(z-z_{0}\right)}\right\} \\
& \cdot \int_{-\infty}^{\infty} \int_{-\infty}^{\infty} d^{2} s d^{2} p \exp \{-\gamma+i k \varphi(\mathbf{s}, \mathbf{p})\},
\end{aligned}
$$

where

$$
\begin{aligned}
& \varphi(\mathbf{s}, \mathbf{p})=2 \mathbf{s}\left(\boldsymbol{\rho}_{+}\left(z_{0}\right)-\boldsymbol{\rho}_{0}\right)+2 \mathbf{p}\left(\boldsymbol{\rho}-\boldsymbol{\rho}_{-}(z)\right) \\
& \quad+2 \int_{z 0}^{z} \mathbf{p}_{-}\left(z^{\prime}\right) \mathbf{p}_{+}\left(z^{\prime}\right) d z^{\prime} \\
& \quad+0.5 \int_{z 0}^{z} \widetilde{\varepsilon}\left[\boldsymbol{\rho}_{+}\left(z^{\prime}\right)+\boldsymbol{\rho}_{-}\left(z^{\prime}\right), z^{\prime}\right] d z^{\prime}
\end{aligned}
$$

$$
\begin{aligned}
& \gamma=\frac{1}{8} \\
& \cdot \int_{z 0}^{z} \frac{\partial^{2}}{\partial \rho_{+}^{2}} \widetilde{\varepsilon}\left[\boldsymbol{\rho}_{+}\left(z^{\prime}\right)+\boldsymbol{\rho}_{-}\left(z^{\prime}\right), z^{\prime}\right] \frac{\partial}{\partial p} \boldsymbol{\rho}_{-}\left(z^{\prime}\right) d z^{\prime}, \\
& \frac{\partial \mathbf{p}_{+,-}\left(z^{\prime}\right)}{\partial z^{\prime}}=\frac{1}{4} \frac{\partial}{\partial \boldsymbol{\rho}_{+}} \widetilde{\varepsilon}\left[\boldsymbol{\rho}_{+}\left(z^{\prime}\right)+\boldsymbol{\rho}_{-}\left(z^{\prime}\right), z^{\prime}\right], \\
& \frac{\partial \boldsymbol{\rho}_{+,-}\left(z^{\prime}\right)}{\partial z^{\prime}}=\mathbf{p}_{+,-}\left(z^{\prime}\right), \\
& E_{0}(\boldsymbol{\rho}, z)=-A_{0}\left(4 \pi\left(z-z_{0}\right)\right)^{-1} \\
& \cdot \exp \left\{i k\left[z-z_{0}+\frac{0.5\left(\rho-\rho_{0}\right)^{2}}{\left(z-z_{0}\right)}\right]\right\}
\end{aligned}
$$

is a field in the absence of inhomogeneities.

\section{The Perturbation Theory in DWFT Solution}

To use solution (24), ray equations (27) with the initial conditions (22) should be solved. Since the exact solution of ray equations for an arbitrary random field $\widetilde{\varepsilon}(\mathbf{r})$ is unknown, we have to use approximate methods.

Supposing the permittivity fluctuations $\widetilde{\mathcal{E}}(\mathbf{r})$ typical of dispersion $\sigma_{\varepsilon}^{2}=\left\langle\widetilde{\varepsilon}^{2}\right\rangle$ are small, that is, considering

$$
\sigma_{\varepsilon}^{2} \ll 1,
$$

let us use the perturbation theory here.

Assume

$$
\boldsymbol{\rho}_{+,-}\left(z^{\prime}\right)=\overline{\boldsymbol{\rho}}_{+,-}\left(z^{\prime}\right)+\widetilde{\boldsymbol{\rho}}_{+,-}\left(z^{\prime}\right),
$$

where

$$
\left|\widetilde{\boldsymbol{\rho}}_{+,-}\left(z^{\prime}\right)\right|=O\left(\sigma_{\varepsilon}\right) .
$$

$\overline{\boldsymbol{\rho}}_{+,-}\left(z^{\prime}\right)$ are solutions of ray equations (27) and (22) in the absence of inhomogeneities $(\widetilde{\varepsilon}=0)$, and the solutions have the form

$$
\begin{aligned}
\overline{\boldsymbol{\rho}}_{+}\left(z^{\prime}\right) & =\mathbf{p}\left(z^{\prime}-z\right), \\
\overline{\boldsymbol{\rho}}_{-}\left(z^{\prime}\right) & =\mathbf{s}\left(z^{\prime}-z_{0}\right), \\
\overline{\boldsymbol{\rho}}\left(\mathbf{p}, \mathbf{s}, z^{\prime}\right) & =\overline{\boldsymbol{\rho}}_{+}\left(z^{\prime}\right)+\overline{\boldsymbol{\rho}}_{-}\left(z^{\prime}\right) \\
& =\mathbf{p}\left(z^{\prime}-z\right)+\mathbf{s}\left(z^{\prime}-z_{0}\right) .
\end{aligned}
$$

For the trajectory variation $\widetilde{\boldsymbol{\rho}}_{+,-}\left(z^{\prime}\right)$, there are equations

$$
\begin{aligned}
& \frac{\partial \widetilde{\mathbf{p}}_{+,-}\left(z^{\prime}\right)}{\partial z^{\prime}}=\frac{1}{4} \frac{\partial}{\partial \boldsymbol{\rho}} \widetilde{\varepsilon}\left[\overline{\boldsymbol{\rho}}\left(z^{\prime}\right)+\widetilde{\boldsymbol{\rho}}_{+}\left(z^{\prime}\right)+\widetilde{\boldsymbol{\rho}}_{-}\left(z^{\prime}\right), z^{\prime}\right], \\
& \frac{\partial \widetilde{\boldsymbol{\rho}}_{+,-}\left(z^{\prime}\right)}{\partial z^{\prime}}=\widetilde{\mathbf{p}}_{+,-}\left(z^{\prime}\right),
\end{aligned}
$$


with zero initial conditions

$$
\widetilde{\boldsymbol{\rho}}_{+}(z)=\widetilde{\boldsymbol{\rho}}_{-}\left(z_{0}\right)=\widetilde{\mathbf{p}}_{+}(z)=\widetilde{\mathbf{p}}_{-}\left(z_{0}\right)=0 .
$$

Use iterations to find approximate solutions (33)-(34):

$$
\begin{aligned}
\widetilde{\boldsymbol{\rho}}_{+}\left(z^{\prime}\right) & =\frac{1}{4} \int_{z}^{z^{\prime}}\left(z^{\prime}-z^{\prime \prime}\right) \frac{\partial}{\partial \boldsymbol{\rho}} \widetilde{\mathcal{\varepsilon}}\left[\overline{\boldsymbol{\rho}}\left(z^{\prime \prime}\right), z^{\prime}\right] d z^{\prime \prime}, \\
\widetilde{\boldsymbol{\rho}}_{-}\left(z^{\prime}\right) & =\frac{1}{4} \int_{z_{0}}^{z^{\prime}}\left(z^{\prime}-z^{\prime \prime}\right) \frac{\partial}{\partial \boldsymbol{\rho}} \widetilde{\mathcal{\varepsilon}}\left[\overline{\boldsymbol{\rho}}\left(z^{\prime \prime}\right), z^{\prime}\right] d z^{\prime \prime}, \\
\tilde{\boldsymbol{\rho}}\left(z^{\prime}\right) & =\widetilde{\boldsymbol{\rho}}_{+}\left(z^{\prime}\right)+\widetilde{\boldsymbol{\rho}}_{-}\left(z^{\prime}\right) \\
& =\frac{1}{4} \int_{z_{0}}^{z}\left|z^{\prime}-z^{\prime \prime}\right| \frac{\partial}{\partial \boldsymbol{\rho}} \widetilde{\mathcal{\varepsilon}}\left[\overline{\boldsymbol{\rho}}\left(z^{\prime \prime}\right), z^{\prime}\right] d z^{\prime \prime} .
\end{aligned}
$$

Inserting (30), (32), and (35) in (24)-(27) and considering that $\gamma=O\left(\widetilde{\varepsilon}^{2}\right)$, we obtain

$$
\begin{aligned}
& E(\boldsymbol{\rho}, z)=E_{0}(\boldsymbol{\rho}, z)\left[\frac{k\left(z-z_{0}\right)}{\pi}\right]^{2} \exp \left\{\frac{i k 2 \boldsymbol{\rho} \boldsymbol{\rho}_{0}}{\left(z-z_{0}\right)}\right\} \\
& \cdot \int_{-\infty}^{\infty} \int_{-\infty}^{\infty} d^{2} s d^{2} p \\
& \quad \cdot \exp \left\{i k\left[\bar{\varphi}(\mathbf{s}, \mathbf{p})+\widetilde{\varphi}(\mathbf{s}, \mathbf{p})+\widetilde{\varphi}_{a}(\mathbf{s}, \mathbf{p})\right]\right\},
\end{aligned}
$$

where

$$
\begin{aligned}
& \bar{\varphi}(\mathbf{s}, \mathbf{p})=-2 \mathbf{p s}\left(z-z_{0}\right)-2 \mathbf{s} \boldsymbol{\rho}_{0}+2 \mathbf{p} \boldsymbol{\rho} \\
& \widetilde{\varphi}(\mathbf{s}, \mathbf{p})=0.5 \int_{z 0}^{z} \widetilde{\varepsilon}\left[\overline{\boldsymbol{\rho}}\left(z^{\prime}\right)+\widetilde{\boldsymbol{\rho}}\left(z^{\prime}\right), z^{\prime}\right] d z^{\prime}, \\
& \varphi_{a}(\mathbf{s}, \mathbf{p}) \\
& \quad=-0.25 \int_{z_{0}}^{z} \widetilde{\boldsymbol{\rho}}\left(z^{\prime}\right) \frac{\partial}{\partial \boldsymbol{\rho}} \widetilde{\mathcal{\varepsilon}}\left[\overline{\boldsymbol{\rho}}\left(z^{\prime}\right)+\widetilde{\boldsymbol{\rho}}\left(z^{\prime}\right), z^{\prime}\right] d z^{\prime}
\end{aligned}
$$

If trajectory variations are less than the minimum size of inhomogeneities

$$
\widetilde{\boldsymbol{\rho}}\left(z^{\prime}\right)<l_{\varepsilon \min }
$$

in (38), we can expand the function $\widetilde{\varepsilon}\left[\overline{\boldsymbol{\rho}}\left(z^{\prime}\right)+\widetilde{\boldsymbol{\rho}}\left(z^{\prime}\right), z^{\prime}\right]$ in powers $\widetilde{\rho}\left(z^{\prime}\right)$ and get

$$
\begin{gathered}
\widetilde{\varphi}(\mathbf{s}, \mathbf{p})+\widetilde{\varphi}_{a}(\mathbf{s}, \mathbf{p})=\frac{1}{2} \int_{z 0}^{z} \widetilde{\mathcal{\varepsilon}}\left[\overline{\boldsymbol{\rho}}\left(z^{\prime}\right), z^{\prime}\right] d z^{\prime}+\frac{1}{8} \\
\cdot \int_{z_{0}}^{z} \frac{\partial}{\partial \boldsymbol{\rho}} \widetilde{\mathcal{\varepsilon}}\left[\overline{\boldsymbol{\rho}}\left(z^{\prime}\right), z^{\prime}\right] \\
\cdot \int_{z_{0}}^{z^{\prime}}\left(z^{\prime}-z^{\prime \prime}\right) \frac{\partial}{\partial \boldsymbol{\rho}} \widetilde{\mathcal{\varepsilon}}\left[\overline{\boldsymbol{\rho}}\left(z^{\prime \prime}\right), z^{\prime}\right] d z^{\prime \prime} d z^{\prime}
\end{gathered}
$$

Taking (41) into account, expression (36) gives the second DWFT approximation, in the integrand of which the terms $O\left(|\widetilde{\varepsilon}|^{3}\right)$ are discarded in phase and $O\left(|\widetilde{\varepsilon}|^{2}\right)$ in the amplitude, respectively.
Compare this approximation to the second GO approximation. To do this, we calculate (36), using the stationary phase method, assuming for simplicity that $\boldsymbol{\rho}=\boldsymbol{\rho}_{0}=0$ :

$$
\begin{aligned}
& E(0, z)=E_{0}(0, z)\left[1+0.25 \int_{z_{0}}^{z}\left(z-z^{\prime}\right)\left(z^{\prime}\right.\right. \\
& \left.\left.\quad-z_{0}\right)\left.\frac{\partial^{2}}{\partial \rho^{2}} \widetilde{\varepsilon}\left[\rho, z^{\prime}\right]\right|_{\rho=0} d z^{\prime}\right]^{-1} \\
& \quad \cdot \exp \left\{i k \varphi_{c}\right\}
\end{aligned}
$$

where

$$
\begin{gathered}
\varphi_{c}=0.5 \int_{z_{0}}^{z} \widetilde{\varepsilon}\left[0, z^{\prime}\right] d z^{\prime}+\left.\frac{1}{4} \int_{z_{0}}^{z} \frac{\partial}{\partial \boldsymbol{\rho}} \widetilde{\varepsilon}\left[\boldsymbol{\rho}, z^{\prime}\right]\right|_{\rho=0} \widetilde{\boldsymbol{\rho}}_{g o}\left(z^{\prime}\right) d z^{\prime} \\
\tilde{\boldsymbol{\rho}}_{g o}\left(z^{\prime}\right)=\frac{-1}{2\left(z-z_{0}\right)}\left[\left(z-z^{\prime}\right)\right. \\
\left.\cdot \int_{z_{0}}^{z^{\prime}}\left(z^{\prime \prime}-z_{0}\right) \frac{\partial}{\partial \boldsymbol{\rho}} \widetilde{\varepsilon}\left[\boldsymbol{\rho}, z^{\prime \prime}\right]\right|_{\boldsymbol{\rho}=0} d z^{\prime \prime}+\left(z^{\prime}-z_{0}\right) \\
\left.\left.\cdot \int_{z^{\prime}}^{z}\left(z-z^{\prime \prime}\right) \frac{\partial}{\partial \boldsymbol{\rho}} \widetilde{\varepsilon}\left[\boldsymbol{\rho}, z^{\prime \prime}\right]\right|_{\boldsymbol{\rho}=0} d z^{\prime \prime}\right]
\end{gathered}
$$

Exactly the same expression was obtained in the GO approximation in [31]. The first and second terms on the right-hand side of (43) describe the phase path variations caused by a phase velocity change and by a trajectory variation, respectively. Typically, in statistical problems the first approximation is used, and the next approximation is calculated mostly to determine the validity range of the first approximation [4]. However, in some problems, estimation of the second approximation is of great importance. For example, in global satellite navigation systems, the first approximation describes a firstorder error that is eliminated in dual-frequency measurements, and the error in these measurements is determined by the next approximation [31].

From derivation of second GO approximation (42), we can see that, for its applicability, the condition of stationary phase method applicability should be met. This condition goes to the condition of the excess of sizes of inhomogeneities over the Fresnel radius

$$
r_{\mathrm{fr}}=\frac{\left(z-z^{\prime}\right)\left(z^{\prime}-z_{0}\right)}{\left[k\left(z-z_{0}\right)\right]}<l_{\varepsilon} .
$$

In addition, condition (40) that sizes of inhomogeneities exceed the trajectory variations should be fulfilled (see also [1]).

\section{Statistical Characteristics of the Wave Field in the First DWFT Approximation}

In the first approximation, when condition (40) is met, the DWFT solution is

$$
\begin{aligned}
& E(\boldsymbol{\rho}, z)=E_{0}(\boldsymbol{\rho}, z)\left[\frac{k\left(z-z_{0}\right)}{\pi}\right]^{2} \exp \left\{\frac{i k 2 \boldsymbol{\rho} \boldsymbol{\rho}_{0}}{\left(z-z_{0}\right)}\right\} \\
& \quad \cdot \int_{-\infty}^{\infty} \int_{-\infty}^{\infty} d^{2} s d^{2} p \\
& \cdot \exp \left\{i 2 k\left[-\mathbf{p s}\left(z-z_{0}\right)-\mathbf{s} \boldsymbol{\rho}_{0}+\mathbf{p} \boldsymbol{\rho}\right]+i k \tilde{\varphi}(\mathbf{s}, \mathbf{p})\right\},
\end{aligned}
$$


where

$$
\begin{aligned}
\widetilde{\varphi}(\mathbf{s}, \mathbf{p}) & =0.5 \int_{z 0}^{z} \widetilde{\varepsilon}\left[\overline{\boldsymbol{\rho}}\left(z^{\prime}\right), z^{\prime}\right] d z^{\prime}, \\
\overline{\boldsymbol{\rho}}\left(z^{\prime}\right) & =\mathbf{p}\left(z^{\prime}-z\right)+\mathbf{s}\left(z^{\prime}-z_{0}\right) .
\end{aligned}
$$

Let us consider $\widetilde{\varepsilon}(\rho, z)$ a real normal quasi-homogeneous random field with a zero mean and correlation function

$$
\begin{aligned}
\Psi_{\varepsilon}\left(\boldsymbol{\rho}_{1}, \boldsymbol{\rho}_{2}, z_{1}, z_{2}\right) & =\left\langle\widetilde{\varepsilon}\left(\boldsymbol{\rho}_{1}, z_{1}\right) \widetilde{\varepsilon}\left(\boldsymbol{\rho}_{2}, z_{2}\right)\right\rangle \\
& =\Psi_{\varepsilon}\left(\Delta \boldsymbol{\rho}, \xi, \boldsymbol{\rho}_{\eta}, \eta\right),
\end{aligned}
$$

where $\Delta \boldsymbol{\rho}=\boldsymbol{\rho}_{1}-\boldsymbol{\rho}_{2}, \boldsymbol{\rho}_{\eta}=\left(\boldsymbol{\rho}_{1}+\boldsymbol{\rho}_{2}\right) / 2, \xi=z_{1}-z_{2}, \eta=$ $\left(z_{1}+z_{2}\right) / 2$.

With respect to the $\widetilde{\varepsilon}(\rho, z)$ field quasi-homogeneity, the first two variables of the function $\Psi_{\varepsilon}\left(\Delta \rho, \xi, \rho_{\eta}, \eta\right)$ change much faster than the others.

4.1. Mean Field. After averaging (46), we get

$$
\begin{aligned}
& \langle E(\boldsymbol{\rho}, z)\rangle=E_{0}(\boldsymbol{\rho}, z)\left[\frac{k\left(z-z_{0}\right)}{\pi}\right]^{2} \exp \left\{\frac{i k 2 \boldsymbol{\rho} \boldsymbol{\rho}_{0}}{\left(z-z_{0}\right)}\right\} \\
& \cdot \int_{-\infty}^{\infty} \int_{-\infty}^{\infty} d^{2} s d^{2} p \\
& \cdot \exp \left\{i 2 k\left[-\mathbf{p s}\left(z-z_{0}\right)-\mathbf{s} \boldsymbol{\rho}_{0}+\mathbf{p} \boldsymbol{\rho}\right]-\frac{\sigma_{\varphi}^{2}}{2}\right\},
\end{aligned}
$$

where

$$
\begin{aligned}
\sigma_{\varphi}^{2} & =0.25 k^{2} \int_{z_{0}}^{z} \bar{\Psi}_{\varepsilon}(0, \eta) d \eta, \\
\bar{\Psi}_{\varepsilon}(\boldsymbol{\rho}(\eta), \eta) & =\int_{-\infty}^{\infty} \Psi_{\varepsilon}\left(\boldsymbol{\rho}(\xi, \eta), \xi, \boldsymbol{\rho}_{\eta}(\xi, \eta), \eta\right) d \xi \\
& \approx \int_{-\infty}^{\infty} \Psi_{\varepsilon}\left(\boldsymbol{\rho}(0, \eta), \xi, \boldsymbol{\rho}_{\eta}(0, \eta), \eta\right) d \xi .
\end{aligned}
$$

Here we take into account the fact that the size of an inhomogeneous area exceeds the correlation radius greatly and use the delta-correlation $\widetilde{\varepsilon}(\rho, z)$ (see [2], for example), which is related to the fact that in the small-angle approximation when integrating (51) we can neglect the $\boldsymbol{\rho}(\xi, \eta)$ dependence on $\xi$.

Considering the absence of the $\sigma_{\varphi}^{2}$ dependence on $\mathbf{s}, \mathbf{p}$, from (49), we obtain

$$
\langle E(\boldsymbol{\rho}, z)\rangle=E_{0}(\boldsymbol{\rho}, z) \exp \left\{\frac{-\sigma_{\varphi}^{2}}{2}\right\} .
$$

This result for the mean field was derived earlier in the DWFT approximation for the 2D-inhomogeneous medium in [26]. It can also be obtained in the Markov and first phase (i.e., when amplitude fluctuations are neglected) GO approximations [2].
4.2. Two-Frequency Mutual Coherence Function. The twofrequency mutual coherence function for the two spherical waves is derived as follows:

$$
\begin{aligned}
\Gamma_{E} & \left(\boldsymbol{\rho}_{1}, \boldsymbol{\rho}_{2}, \omega_{1}, \omega_{2}\right) \\
\quad & \left\langle E\left(\boldsymbol{\rho}_{1}, \omega_{1}, z, z_{0}\right) E^{*}\left(\boldsymbol{\rho}_{2}, \omega_{2}, z, z_{0}\right)\right\rangle .
\end{aligned}
$$

By substituting (46) in (53) and averaging, we get

$$
\begin{aligned}
\Gamma_{E} & \left(\boldsymbol{\rho}_{1}, \boldsymbol{\rho}_{2}, \omega_{1}, \omega_{2}\right) \\
\quad & =E_{0}\left(\boldsymbol{\rho}_{1}, \omega_{1}\right) E_{0}{ }^{*}\left(\boldsymbol{\rho}_{2}, \omega_{2}\right) \widehat{\Gamma}_{2}\left(\boldsymbol{\rho}_{1}, \boldsymbol{\rho}_{2}, \omega_{1}, \omega_{2}\right),
\end{aligned}
$$

where

$$
\begin{aligned}
& \widehat{\Gamma}_{2}\left(\boldsymbol{\rho}_{1}, \boldsymbol{\rho}_{2}, \omega_{1}, \omega_{2}\right)=\left\langle W_{2}\left(\boldsymbol{\rho}_{1}, \boldsymbol{\rho}_{2}, \omega_{1}, \omega_{2}\right)\right\rangle, \\
& W_{2}\left(\boldsymbol{\rho}_{1}, \boldsymbol{\rho}_{2}, \omega_{1}, \omega_{2}\right)=\left(k_{1} k_{2}\right)^{2}\left[\frac{\left(z-z_{0}\right)}{\pi}\right]^{4} \\
& \cdot \exp \left\{\frac{i 2\left(k_{1} \boldsymbol{\rho}_{1} \boldsymbol{\rho}_{01}-k_{2} \boldsymbol{\rho}_{2} \boldsymbol{\rho}_{02}\right)}{\left(z-z_{0}\right)}\right\} \\
& \cdot \int_{-\infty}^{\infty} \cdots \int_{-\infty}^{\infty} d^{2} s_{1} \cdots d^{2} p_{2} \\
& \cdot \exp \left\{-i 2 k_{1}\left[\mathbf{p}_{1} \mathbf{s}_{1}\left(z-z_{0}\right)+\mathbf{s}_{1} \boldsymbol{\rho}_{01}-\mathbf{p}_{1} \boldsymbol{\rho}_{1}\right]\right. \\
& \quad+i 2 k_{1}\left[\mathbf{p}_{2} \mathbf{s}_{2}\left(z-z_{0}\right)+\mathbf{s}_{2} \boldsymbol{\rho}_{02}-\mathbf{p}_{2} \boldsymbol{\rho}_{2}\right] \\
& \left.\quad+i k_{1} \widetilde{\varphi}\left(\mathbf{s}_{1}, \mathbf{p}_{1}\right)-i k_{2} \widetilde{\varphi}\left(\mathbf{s}_{2}, \mathbf{p}_{2}\right)\right\} .
\end{aligned}
$$

After averaging (56), we obtain

$$
\begin{aligned}
& \widehat{\Gamma}_{2}\left(\boldsymbol{\rho}_{1}, \boldsymbol{\rho}_{2}, \omega_{1}, \omega_{2}\right)=\left(k_{1} k_{2}\right)^{2}\left[\frac{\left(z-z_{0}\right)}{\pi}\right]^{4} \\
& \cdot \exp \left\{\frac{i 2\left(k_{1} \boldsymbol{\rho}_{1} \boldsymbol{\rho}_{01}-k_{2} \boldsymbol{\rho}_{2} \boldsymbol{\rho}_{02}\right)}{\left(z-z_{0}\right)}\right\} \int_{-\infty}^{\infty} \cdots \int_{-\infty}^{\infty} d^{2} s_{1} \\
& \quad \cdots d^{2} p_{2} \exp \left\{-i 2 k_{1}\left[\mathbf{p}_{1} \mathbf{s}_{1}\left(z-z_{0}\right)+\mathbf{s}_{1} \boldsymbol{\rho}_{01}-\mathbf{p}_{1} \boldsymbol{\rho}_{1}\right]\right. \\
& \left.+i 2 k_{1}\left[\mathbf{p}_{2} \mathbf{s}_{2}\left(z-z_{0}\right)+\mathbf{s}_{2} \boldsymbol{\rho}_{02}-\mathbf{p}_{2} \boldsymbol{\rho}_{2}\right]-\frac{D_{2 \varphi}}{2}\right\} \\
& D_{2 \varphi}=0.25 \int_{z_{0}}^{z}\left[\left(k_{1}^{2}+k_{2}^{2}\right) \bar{\Psi}_{\varepsilon}(0, \eta)\right. \\
& \quad-2 k_{1} k_{2} \bar{\Psi}_{\varepsilon}\left(\left(\mathbf{p}_{1}-\mathbf{p}_{2}\right)\left(z^{\prime}-z\right)\right. \\
& \left.\left.+\left(\mathbf{s}_{1}-\mathbf{s}_{2}\right)\left(z^{\prime}-z_{0}\right), \eta\right)\right] d \eta .
\end{aligned}
$$

Substituting integration variables in (57) $\mathbf{s}_{1,2}=\overline{\mathbf{s}} \pm \mathbf{s} / 2, \mathbf{p}_{1,2}=$ $\overline{\mathbf{p}} \pm \mathbf{p} / 2$ and taking integrals over $\overline{\mathbf{s}}$ and $\overline{\mathbf{p}}$, we get

$$
\begin{aligned}
& \widehat{\Gamma}_{2}\left(\Delta \boldsymbol{\rho}, \Delta \boldsymbol{\rho}_{0}, k_{0}, X\right)=\left[\frac{\widehat{k}_{0}\left(z-z_{0}\right)}{(2 \pi)}\right]^{2} \\
& \cdot \exp \left\{i \widehat{k}_{0} \Delta \boldsymbol{\rho}, \frac{\Delta \boldsymbol{\rho}_{0}}{\left(z-z_{0}\right)}\right\} \int_{-\infty}^{\infty} \int_{-\infty}^{\infty} d^{2} s d^{2} p
\end{aligned}
$$




$$
\cdot \exp \left\{-i \bar{k}_{0}\left[\mathbf{p s}\left(z-z_{0}\right)+\mathbf{s} \Delta \boldsymbol{\rho}_{0}-\mathbf{p} \Delta \boldsymbol{\rho}\right]-\frac{\widehat{D}_{2 \varphi}}{2}\right\}
$$

Here

$$
\begin{aligned}
\widehat{D}_{2 \varphi} & =0.5 k_{0}^{2} \int_{z_{0}}^{z}\left[\left(1+X^{2}\right) \bar{\Psi}_{\varepsilon}(0, \eta)\right. \\
- & \left.\left(1-X^{2}\right) \bar{\Psi}_{\varepsilon}(\overline{\boldsymbol{\rho}}(\mathbf{p}, \mathbf{s}, \eta), \eta)\right] d \eta
\end{aligned}
$$

and the notations are introduced (see [19], for example):

$$
\begin{aligned}
& k_{0}=\frac{\left(k_{1}+k_{2}\right)}{2}, \\
& X=\frac{\left(k_{2}-k_{1}\right)}{2 k_{0}}, \\
& \hat{k}_{0}=k_{0}\left(X^{-1}-X\right),
\end{aligned}
$$

$$
\Delta \rho_{0}=\rho_{01}-\rho_{02}
$$

A spatial coherence function can be obtained from twofrequency mutual coherence function (53), (59)-(60) by setting the frequency separation $X$ to zero:

$$
\begin{aligned}
& \Gamma_{E}\left(\Delta \boldsymbol{\rho}, \Delta \boldsymbol{\rho}_{0}\right)=E_{0}\left(\boldsymbol{\rho}_{1}\right) E_{0}{ }^{*}\left(\boldsymbol{\rho}_{2}\right) \exp \left\{-\frac{k^{2}}{4}\right. \\
& \left.\quad \cdot \int_{z_{0}}^{z}\left[\bar{\Psi}_{\varepsilon}(0, \eta)-\bar{\Psi}_{\varepsilon}\left(\frac{\Delta \boldsymbol{\rho}_{0}(z-\eta)}{z-z_{0}}+\frac{\Delta \boldsymbol{\rho}\left(\eta-z_{0}\right)}{z-z_{0}}, \eta\right)\right] d \eta\right\} .
\end{aligned}
$$

Expression (62), like formula (52) for the mean field, coincides with the results of the Markov approximation [2], and it can also be obtained from the phase GO approximation.

For small but finite $X$, when analyzing the narrowband signals propagation, expression (60) can be calculated asymptotically, and from (53) we obtain

$$
\begin{aligned}
\Gamma_{E}\left(\boldsymbol{\rho}_{1}, \boldsymbol{\rho}_{2}, \omega_{1}, \omega_{2}\right) \approx & E_{0}\left(\boldsymbol{\rho}_{1}\right) E_{0}{ }^{*}\left(\boldsymbol{\rho}_{2}\right)\left[1-i S\left(\Delta \boldsymbol{\rho}, \Delta \boldsymbol{\rho}_{0}, k_{0}, X\right)\right]^{-1 / 2} \\
& \cdot \exp \left\{-\frac{k_{0}^{2}}{4} \int_{z_{0}}^{z}\left[\left(1+X^{2}\right) \bar{\Psi}_{\varepsilon}(0, \eta)-\left(1-X^{2}\right) \bar{\Psi}_{\varepsilon}\left(\frac{\Delta \boldsymbol{\rho}_{0}(z-\eta)}{z-z_{0}}+\frac{\Delta \boldsymbol{\rho}\left(\eta-z_{0}\right)}{z-z_{0}}, \eta\right)\right] d \eta\right\}
\end{aligned}
$$

where

$$
S=\frac{X}{4 k_{0}\left(z-z_{0}\right)} \int_{z_{0}}^{z}\left(\eta-z_{0}\right)(z-\eta) \frac{\partial^{2}}{\partial \rho^{2}} \bar{\Psi}_{\varepsilon}\left(\frac{\Delta \boldsymbol{\rho}_{0}(z-\eta)}{z-z_{0}}+\frac{\Delta \boldsymbol{\rho}\left(\eta-z_{0}\right)}{z-z_{0}}, \eta\right) d \eta \text {. }
$$

The condition of applicability of asymptotic expression (63) is

$$
\widehat{r}_{f r}<l_{\varepsilon},
$$

where

$$
\widehat{r}_{f r}=\frac{\left(z-z^{\prime}\right)\left(z^{\prime}-z_{0}\right)}{\left[\widehat{k}_{0}\left(z-z_{0}\right)\right]}=\frac{r_{f r}}{X^{-1}-X}<r_{f r} .
$$

For narrow-band signals, when $X \ll 1$, condition (66) is much weaker than the condition of GO approximation (45).

\section{Second-Order Effects in an Inhomogeneous Medium}

Here we account for the fact that, in inhomogeneous media, with distance from the source, the trajectory fluctuations grow; at a distance great enough, these fluctuations become larger than sizes of some inhomogeneities, and condition (40) that was used above is no more applicable, at least for smallscale inhomogeneities. So here, in (38) we leave the trajectory variations in the argument. On the other hand, using (36), we neglect phase addition $\widetilde{\varphi}_{a}(\mathbf{s}, \mathbf{p})$. Thus, we use an expression similar to (47)

$$
\begin{aligned}
& E(\boldsymbol{\rho}, z)=E_{0}(\boldsymbol{\rho}, z)\left[\frac{k\left(z-z_{0}\right)}{\pi}\right]^{2} \exp \left\{\frac{i k 2 \boldsymbol{\rho} \boldsymbol{\rho}_{0}}{\left(z-z_{0}\right)}\right\} \\
& \cdot \int_{-\infty}^{\infty} \int_{-\infty}^{\infty} d^{2} s d^{2} p \\
& \quad \cdot \exp \left\{-i 2 k\left[\mathbf{p s}\left(z-z_{0}\right)+\mathbf{s} \boldsymbol{\rho}_{0}-\mathbf{p} \boldsymbol{\rho}\right]+i k \widetilde{\varphi}_{d}(\mathbf{s}, \mathbf{p})\right\},
\end{aligned}
$$

but with another phase fluctuation:

$$
\widetilde{\varphi}_{d}(\mathbf{s}, \mathbf{p})=0.5 \int_{z 0}^{z} \widetilde{\mathcal{E}}\left[\overline{\boldsymbol{\rho}}\left(z^{\prime}\right)+\widetilde{\boldsymbol{\rho}}\left(z^{\prime}\right), z^{\prime}\right] d z^{\prime},
$$

where $\overline{\boldsymbol{\rho}}\left(z^{\prime}\right)$ and $\tilde{\boldsymbol{\rho}}\left(z^{\prime}\right)$ are described by expressions (32) and (35), respectively.

As we can see from (68), averaging of expression (67), required to calculate statistical characteristics, is complicated by the nonlinear dependence of $(68)$ on $\widetilde{\varepsilon}(\rho, z)$. To solve this problem, we use a hybrid approach $[5,9]$. We assume that 
the random permittivity field $\widetilde{\mathcal{E}}(\rho, z)$ can be represented as the sum of two independent random fields:

$$
\widetilde{\varepsilon}(\rho, z)=\widetilde{\varepsilon}_{1}(\rho, z)+\widetilde{\varepsilon}_{2}(\rho, z),
$$

where $\widetilde{\varepsilon}_{1}(\rho, z)$ and $\widetilde{\varepsilon}_{2}(\rho, z)$ correspond to large-scale and small-scale inhomogeneities, respectively. With that, we account for the trajectory variations only when estimating the contribution of small-scale inhomogeneities. In this case,

$$
\begin{aligned}
& \widetilde{\varphi}_{d}(\mathbf{s}, \mathbf{p})=\widetilde{\varphi}_{1}(\mathbf{s}, \mathbf{p})+\widetilde{\varphi}_{2}(\mathbf{s}, \mathbf{p}) \\
& \widetilde{\varphi}_{1}(\mathbf{s}, \mathbf{p})=0.5 \int_{z 0}^{z} \widetilde{\varepsilon}_{1}\left[\overline{\boldsymbol{\rho}}\left(z^{\prime}\right), z^{\prime}\right] d z^{\prime} \\
& \widetilde{\varphi}_{2}(\mathbf{s}, \mathbf{p})=0.5 \int_{z 0}^{z} \widetilde{\varepsilon}_{2}\left[\overline{\boldsymbol{\rho}}\left(z^{\prime}\right)+\widetilde{\boldsymbol{\rho}}\left(z^{\prime}\right), z^{\prime}\right] d z^{\prime}
\end{aligned}
$$

Furthermore, we consider the fact that, due to a rapid drop in the inhomogeneities spectrum, the contribution of smallscale inhomogeneities to trajectory variations in (35) can be neglected:

$$
\begin{aligned}
\tilde{\boldsymbol{\rho}}\left(z^{\prime}\right) & \approx \widetilde{\boldsymbol{\rho}}_{1}\left(z^{\prime}\right) \\
& =\frac{1}{4} \int_{z_{0}}^{z}\left|z^{\prime}-z^{\prime \prime}\right| \frac{\partial}{\partial \boldsymbol{\rho}} \widetilde{\varepsilon}_{1}\left[\overline{\boldsymbol{\rho}}\left(z^{\prime \prime}\right), z^{\prime}\right] d z^{\prime \prime} .
\end{aligned}
$$

After substituting (70)-(73) in (67), we get

$$
\begin{aligned}
& E(\boldsymbol{\rho}, z)=E_{0}(\boldsymbol{\rho}, z)\left[\frac{k\left(z-z_{0}\right)}{\pi}\right]^{2} \exp \left\{\frac{i k 2 \boldsymbol{\rho} \boldsymbol{\rho}_{0}}{\left(z-z_{0}\right)}\right\} \\
& \quad \cdot \int_{-\infty}^{\infty} \int_{-\infty}^{\infty} d^{2} s d^{2} p \\
& \quad \cdot \exp \left\{-i 2 k\left[\mathbf{p s}\left(z-z_{0}\right)+\mathbf{s} \boldsymbol{\rho}_{0}-\mathbf{p} \boldsymbol{\rho}\right]+i k \tilde{\varphi}_{1}(\mathbf{s}, \mathbf{p})\right. \\
& \left.+i k \tilde{\varphi}_{2}(\mathbf{s}, \mathbf{p})\right\} .
\end{aligned}
$$

5.1. Mean Field. Since $\widetilde{\varepsilon}_{2}$ is a linear part of exponent argument in (74), being independent of $\widetilde{\varepsilon}_{1}$, averaging (71) only over realizations of $\widetilde{\varepsilon}_{2}$ yields

$$
\begin{aligned}
& \left\langle E(\boldsymbol{\rho}, z) \mid \widetilde{\varepsilon}_{1}\right\rangle=E_{0}(\boldsymbol{\rho}, z)\left[\frac{k\left(z-z_{0}\right)}{\pi}\right]^{2} \\
& \cdot \exp \left\{\frac{i k 2 \boldsymbol{\rho} \boldsymbol{\rho}_{0}}{\left(z-z_{0}\right)}\right\} \int_{-\infty}^{\infty} \int_{-\infty}^{\infty} d^{2} s d^{2} p \\
& \cdot \exp \left\{-i 2 k\left[\mathbf{p s}\left(z-z_{0}\right)+\mathbf{s} \boldsymbol{\rho}_{0}-\mathbf{p} \boldsymbol{\rho}\right]+i k \widetilde{\varphi}_{1}(\mathbf{s}, \mathbf{p})\right. \\
& \left.-\frac{\sigma_{\varphi 2}^{2}}{2}\right\}
\end{aligned}
$$

where, with the delta-correlation of $\widetilde{\varepsilon}_{2}$, just as in derivation of (50), we have

$$
\sigma_{\varphi 2}^{2}=0.25 k^{2} \int_{z_{0}}^{z} \bar{\Psi}_{\varepsilon 2}(0, \eta) d \eta .
$$

After averaging (75) over realizations $\widetilde{\varepsilon}_{1}$, we get

$$
\langle E(\boldsymbol{\rho}, z)\rangle=E_{0}(\boldsymbol{\rho}, z) \exp \left\{-\frac{\sigma_{\varphi}^{2}}{2}\right\},
$$

where

$$
\sigma_{\varphi}^{2}=\sigma_{\varphi 1}^{2}+\sigma_{\varphi 2}^{2}=0.25 k^{2} \int_{z_{0}}^{z} \bar{\Psi}_{\varepsilon}(0, \eta) d \eta .
$$

From the comparison between (77) and (52), it is apparent that the consideration of the incident wave distortions does not affect the mean field.

5.2. Two-Frequency Mutual Coherence Function. Substituting (74) in (55) gives

$$
\begin{aligned}
& W_{2}\left(\boldsymbol{\rho}_{1}, \boldsymbol{\rho}_{2}, \omega_{1}, \omega_{2}\right)=\left(k_{1} k_{2}\right)^{2}\left[\frac{\left(z-z_{0}\right)}{\pi}\right]^{4} \\
& \cdot \exp \left\{\frac{i 2\left(k_{1} \boldsymbol{\rho}_{1} \boldsymbol{\rho}_{01}-k_{2} \boldsymbol{\rho}_{2} \boldsymbol{\rho}_{02}\right)}{\left(z-z_{0}\right)}\right\} \\
& \cdot \int_{-\infty}^{\infty} \cdots \int_{-\infty}^{\infty} d^{2} s_{1} \cdots d^{2} p_{2} \\
& \cdot \exp \left\{-i 2 k_{1}\left[\mathbf{p}_{1} \mathbf{s}_{1}\left(z-z_{0}\right)+\mathbf{s}_{1} \boldsymbol{\rho}_{01}-\mathbf{p}_{1} \boldsymbol{\rho}_{1}\right]\right. \\
& +i 2 k_{1}\left[\mathbf{p}_{2} \mathbf{s}_{2}\left(z-z_{0}\right)+\mathbf{s}_{2} \boldsymbol{\rho}_{02}-\mathbf{p}_{2} \boldsymbol{\rho}_{2}\right] \\
& +i k_{1} \widetilde{\varphi}_{1}\left(\mathbf{s}_{1}, \mathbf{p}_{1}\right)-i k_{2} \widetilde{\varphi}_{1}\left(\mathbf{s}_{2}, \mathbf{p}_{2}\right)+i k_{1} \widetilde{\varphi}_{2}\left(\mathbf{s}_{1}, \mathbf{p}_{1}\right) \\
& \left.-i k_{2} \widetilde{\varphi}_{2}\left(\mathbf{s}_{2}, \mathbf{p}_{2}\right)\right\} .
\end{aligned}
$$

By averaging the resulting expressions over realizations $\widetilde{\varepsilon}_{2}$, we get

$$
\begin{aligned}
& \left\langle W_{2}\left(\boldsymbol{\rho}_{1}, \boldsymbol{\rho}_{2}, \omega_{1}, \omega_{2}\right) \mid \widetilde{\varepsilon}_{1}\right\rangle=\left(k_{1} k_{2}\right)^{2}\left[\frac{\left(z-z_{0}\right)}{\pi}\right]^{4} \\
& \cdot \exp \left\{\frac{i 2\left(k_{1} \boldsymbol{\rho}_{1} \boldsymbol{\rho}_{01}-k_{2} \boldsymbol{\rho}_{2} \boldsymbol{\rho}_{02}\right)}{\left(z-z_{0}\right)}\right\} \\
& \cdot \int_{-\infty}^{\infty} \cdots \int_{-\infty}^{\infty} d^{2} s_{1} \cdots d^{2} p_{2} \\
& \cdot \exp \left\{-i 2 k_{1}\left[\mathbf{p}_{1} \mathbf{s}_{1}\left(z-z_{0}\right)+\mathbf{s}_{1} \boldsymbol{\rho}_{01}-\mathbf{p}_{1} \boldsymbol{\rho}_{1}\right]\right. \\
& +i 2 k_{1}\left[\mathbf{p}_{2} \mathbf{s}_{2}\left(z-z_{0}\right)+\mathbf{s}_{2} \boldsymbol{\rho}_{02}-\mathbf{p}_{2} \boldsymbol{\rho}_{2}\right] \\
& \left.+i k_{1} \widetilde{\varphi}_{1}\left(\mathbf{s}_{1}, \mathbf{p}_{1}\right)-i k_{2} \widetilde{\varphi}_{1}\left(\mathbf{s}_{2}, \mathbf{p}_{2}\right)-\frac{D_{\varphi 2}}{2}\right\}
\end{aligned}
$$

where

$$
\begin{gathered}
D_{\varphi 2}=0.25 \int_{z_{0}}^{z}\left[\left(k_{1}^{2}+k_{2}^{2}\right) \bar{\Psi}_{\varepsilon 2}(0, \eta)\right. \\
\quad-2 k_{1} k_{2} \bar{\Psi}_{\varepsilon 2}\left(\left(\mathbf{p}_{1}-\mathbf{p}_{2}\right)\left(z^{\prime}-z\right)\right. \\
\left.\left.\quad+\left(\mathbf{s}_{1}-\mathbf{s}_{2}\right)\left(z^{\prime}-z_{0}\right), \eta\right)\right] d \eta
\end{gathered}
$$


We take into account the fact that for small-scale inhomogeneities

$$
D_{\varphi 2}<1
$$

and rewrite (80):

$$
\left\langle W_{2}\left(\boldsymbol{\rho}_{1}, \boldsymbol{\rho}_{2}, \omega_{1}, \omega_{2}\right) \mid \widetilde{\varepsilon}_{1}\right\rangle \approx W_{2 d 1}+W_{2 d 2},
$$

where

$$
\begin{aligned}
& W_{2 d 1}=W_{2}\left\{1-0.125 \int_{z_{0}}^{z}\left(k_{1}^{2}+k_{2}^{2}\right) \bar{\Psi}_{\varepsilon 2}\left(0, z_{+}\right) d z_{+}\right\} \\
& W_{2 d 2}=0.25\left(k_{1} k_{2}\right)^{3}\left[\frac{\left(z-z_{0}\right)}{\pi}\right]^{4} \\
& \quad \cdot \exp \left\{\frac{i 2\left(k_{1} \boldsymbol{\rho}_{1} \boldsymbol{\rho}_{01}-k_{2} \boldsymbol{\rho}_{2} \boldsymbol{\rho}_{02}\right)}{\left(z-z_{0}\right)}\right\} \int_{-\infty}^{\infty} \cdots \int_{-\infty}^{\infty} d^{2} s_{1} \\
& \quad \cdots d^{2} p_{2} \int_{z_{0}}^{z} d z^{\prime} \bar{\Psi}_{\varepsilon 2}\left(\left(\mathbf{p}_{1}-\mathbf{p}_{2}\right)\left(z^{\prime}-z\right)\right. \\
& \left.+\left(\mathbf{s}_{1}-\mathbf{s}_{2}\right)\left(z^{\prime}-z_{0}\right), \eta\right) \exp \left\{-i 2 k_{1}\left[\mathbf{p}_{1} \mathbf{s}_{1}\left(z-z_{0}\right)\right.\right. \\
& \left.+\mathbf{s}_{1} \boldsymbol{\rho}_{01}-\mathbf{p}_{1} \boldsymbol{\rho}_{1}\right]+i 2 k_{1}\left[\mathbf{p}_{2} \mathbf{s}_{2}\left(z-z_{0}\right)+\mathbf{s}_{2} \boldsymbol{\rho}_{02}\right. \\
& \left.\left.-\mathbf{p}_{2} \boldsymbol{\rho}_{2}\right]+i k_{1} \widetilde{\varphi}_{1}\left(\mathbf{s}_{1}, \mathbf{p}_{1}\right)-i k_{2} \widetilde{\varphi}_{1}\left(\mathbf{s}_{2}, \mathbf{p}_{2}\right)\right\} .
\end{aligned}
$$

In (84), $W_{2}$ is a function (56) used above to investigate the two-frequency mutual coherence function, without regard to second-order effects for $\widetilde{\varepsilon}=\widetilde{\varepsilon}_{1}$.

When calculating (85), we have to take into account the relationship of the spectrum and the correlation function

$$
\begin{aligned}
& \bar{\Psi}_{\varepsilon 2}\left(\boldsymbol{\rho}\left(z_{+}\right), z_{+}\right) \\
& \quad=2 \pi \int_{-\infty}^{\infty} d^{2} \kappa \Phi\left(\boldsymbol{\kappa}, z_{+}\right) \exp \left[i \boldsymbol{\kappa} \boldsymbol{\rho}\left(z_{+}\right)\right] .
\end{aligned}
$$

Besides, considering the smoothness of large-scale inhomogeneities, we assume

$$
\begin{aligned}
k \widetilde{\varphi}_{1}\left(\mathbf{p}_{1}, \mathbf{s}_{1}\right)+\boldsymbol{\kappa} \widetilde{\boldsymbol{\rho}}\left(\mathbf{p}_{1}, \mathbf{s}_{1}, z_{+}\right) & \\
= & 0.5 k \int_{z 0}^{z} \widetilde{\varepsilon}_{1}\left[\overline{\boldsymbol{\rho}}\left(\mathbf{p}_{1}, \mathbf{s}_{1}, \eta\right), \eta\right] d \eta \\
& +\frac{\boldsymbol{\kappa}}{4} \int_{z_{0}}^{z}\left|z_{+}-\eta\right| \frac{\partial}{\partial \boldsymbol{\rho}} \widetilde{\varepsilon}_{1}\left[\overline{\boldsymbol{\rho}}\left(\mathbf{p}_{1}, \mathbf{s}_{1}, \eta\right), \eta\right] d \eta \\
\approx & 0.5 k \int_{z 0}^{z} \widetilde{\varepsilon}_{1}\left[\overline{\boldsymbol{\rho}}\left(\mathbf{p}_{1}, \mathbf{s}_{1}, \eta\right)+\frac{\boldsymbol{\kappa}}{2 k}\left|z_{+}-\eta\right|, \eta\right] d \eta .
\end{aligned}
$$

As a result, by substituting (86)-(87) in (85), we obtain

$$
\begin{gathered}
W_{2 d 2}=0.5 \pi\left(k_{1} k_{2}\right)^{3}\left[\frac{\left(z-z_{0}\right)}{\pi}\right]^{4} \\
\cdot \exp \left\{\frac{i 2\left(k_{1} \boldsymbol{\rho}_{1} \boldsymbol{\rho}_{01}-k_{2} \boldsymbol{\rho}_{2} \boldsymbol{\rho}_{02}\right)}{\left(z-z_{0}\right)}\right\} \\
\cdot \int_{-\infty}^{\infty} \int_{-\infty}^{\infty} \int_{z_{0}}^{z} d z^{\prime} d^{2} s_{1} d^{2} p_{1} d^{2} s_{2} d^{2} p_{2}
\end{gathered}
$$

$$
\begin{aligned}
& \cdot \int_{-\infty}^{\infty} d^{2} \kappa \Phi_{\varepsilon 2}\left(\boldsymbol{\kappa}, z_{+}\right) \\
& \cdot \exp \left\{i \boldsymbol{\kappa}\left[\overline{\boldsymbol{\rho}}\left(\mathbf{s}_{1}, \mathbf{p}_{1}, z_{+}\right)-\overline{\boldsymbol{\rho}}\left(\mathbf{s}_{2}, \mathbf{p}_{2}, z_{+}\right)\right]\right. \\
& -i 2 k_{1}\left[\mathbf{p}_{1} \mathbf{s}_{1}\left(z-z_{0}\right)+\mathbf{s}_{1} \boldsymbol{\rho}_{01}-\mathbf{p}_{1} \boldsymbol{\rho}_{1}\right] \\
& +i 2 k_{1}\left[\mathbf{p}_{2} \mathbf{s}_{2}\left(z-z_{0}\right)+\mathbf{s}_{2} \boldsymbol{\rho}_{02}-\mathbf{p}_{2} \boldsymbol{\rho}_{2}\right] \\
& +i 0.5 k_{1} \int_{z 0}^{z} \widetilde{\varepsilon}_{1}\left[\overline{\boldsymbol{\rho}}\left(\mathbf{p}_{1}, \mathbf{s}_{1}, \eta\right)+\frac{\boldsymbol{\kappa}}{2 k_{1}}\left|z_{+}-\eta\right|, \eta\right] d \eta \\
& \left.-i 0.5 k_{2} \int_{z 0}^{z} \widetilde{\varepsilon}_{1}\left[\overline{\boldsymbol{\rho}}\left(\mathbf{p}_{2}, \mathbf{s}_{2}, \eta\right)+\frac{\boldsymbol{\kappa}}{2 k_{2}}\left|z_{+}-\eta\right|, \eta\right] d \eta\right\} .
\end{aligned}
$$

We average (88) over large-scale fluctuations $\widetilde{\varepsilon}_{1}$ and turn to variables $\mathbf{s}_{1,2}=\overline{\mathbf{s}} \pm \mathbf{s} / 2, \mathbf{p}_{1,2}=\overline{\mathbf{p}} \pm \mathbf{p} / 2$ and evaluate integrals over $\overline{\mathbf{s}}$ and $\overline{\mathbf{p}}$. After summing up with $W_{2 d 1}$, we get

$$
\begin{aligned}
\widehat{\Gamma}_{2} & \left(\boldsymbol{\rho}_{1}, \boldsymbol{\rho}_{2}, \omega_{1}, \omega_{2}\right)=\left\langle W_{2}\left(\boldsymbol{\rho}_{1}, \boldsymbol{\rho}_{2}, \omega_{1}, \omega_{2}\right)\right\rangle \\
= & {\left[\frac{\hat{k}_{0}\left(z-z_{0}\right)}{2 \pi}\right]^{2} \exp \left\{i \hat{k}_{0} \Delta \boldsymbol{\rho}, \frac{\Delta \boldsymbol{\rho}_{0}}{\left(z-z_{0}\right)}\right\} } \\
\cdot & \int_{-\infty}^{\infty} \int_{-\infty}^{\infty} d^{2} s d^{2} p \\
\cdot & \exp \left\{-i \widehat{k}_{0}\left[\mathbf{p s}\left(z-z_{0}\right)+\mathbf{s} \Delta \boldsymbol{\rho}_{0}-\mathbf{p} \Delta \boldsymbol{\rho}\right]\right\} \\
\cdot & \left\{\operatorname { e x p } [ \frac { - \widehat { D } _ { 1 B } ( \mathbf { p } , \mathbf { s } , 0 ) } { 2 } ] \left\{1-\frac{k_{0}^{2}}{4}\left(1+X^{2}\right)\right.\right. \\
\cdot & \left.\int_{z_{0}}^{z} \bar{\Psi}_{\varepsilon 2}\left(0, z_{+}\right) d z_{+}\right\}+\pi \frac{k_{0}^{2}}{2}\left(1-X^{2}\right) \\
\cdot & \int_{z_{0}}^{z} d z_{+} \int_{-\infty}^{\infty} d^{2} \kappa \Phi_{\varepsilon 2}\left(\boldsymbol{\kappa}, z_{+}\right) \\
\cdot & \left.\exp \left[i \boldsymbol{\kappa} \overline{\boldsymbol{\rho}}\left(\mathbf{s}, \mathbf{p}, z_{+}\right)-\frac{D_{1 B}(\mathbf{p}, \mathbf{s}, \boldsymbol{\kappa})}{2}\right]\right\},
\end{aligned}
$$

where

$$
\begin{aligned}
& \widehat{D}_{1 B}(\mathbf{p}, \mathbf{s}, \boldsymbol{\kappa})=0.5 k_{0}^{2} \int_{z_{0}}^{z}\left[\left(1+X^{2}\right) \bar{\Psi}_{\varepsilon 1}(0, \eta)\right. \\
& -\left(1-X^{2}\right) \\
& \left.. \bar{\Psi}_{\varepsilon 1}\left(\overline{\boldsymbol{\rho}}(\mathbf{p}, \mathbf{s}, \eta)+\frac{0.5 \boldsymbol{\kappa}\left|z_{+}-\eta\right|}{\widehat{k}_{0}}, \eta\right)\right] d \eta .
\end{aligned}
$$

If we assume

$$
\frac{\boldsymbol{\kappa}\left|z_{+}-\eta\right|}{\left(\widehat{k}_{0} l_{\varepsilon}\right)} \ll 1,
$$

that is, neglect the dependence of (90) on $\boldsymbol{\kappa}$, from (89), we get previously obtained expression (57). This means that when condition (91) is met, the second-order effects, which 
are related to the front curvature of a wave incident on an inhomogeneity, are not significant in the two-frequency mutual coherence function.

Note that, with the bandwidth decreasing, $\widehat{k}_{0}$ grows, and for $\Delta \omega=\omega_{2}-\omega_{1}=0$, that is, for a monochrome signal, conditions (91) are always met. It follows that the second-order effects do not affect the behavior of the spatial correlation function.

5.3. Intensity Spatial Correlation. As the previous paragraph shows, the second-order effects are not significant in the behavior of the field spatial correlation function. Now let us consider the spatial correlation function for intensity.

As the intensity equals $I(\mathbf{r})=|E(\mathbf{r})|^{2}$, the correlation function for intensity equals

$$
\begin{aligned}
& \Psi_{I}\left(\boldsymbol{\rho}_{1}, \boldsymbol{\rho}_{2}, z\right) \\
& \quad=\left\langle\left[I\left(\boldsymbol{\rho}_{1}, z\right)-\left\langle I\left(\boldsymbol{\rho}_{1}, z\right)\right\rangle\right]\left[I\left(\boldsymbol{\rho}_{2}, z\right)-\left\langle I\left(\boldsymbol{\rho}_{2}, z\right)\right\rangle\right]\right\rangle \\
& =\left\langle I\left(\boldsymbol{\rho}_{1}, z\right) I\left(\boldsymbol{\rho}_{2}, z\right)\right\rangle-\left\langle I\left(\boldsymbol{\rho}_{1}, z\right)\right\rangle\left\langle I\left(\boldsymbol{\rho}_{2}, z\right)\right\rangle .
\end{aligned}
$$

From (62), it follows that $\langle I(\rho, z)\rangle=\left\langle|U(\rho, z)|^{2}\right\rangle=\Gamma_{E}(0,0)=$ $\left|E_{0}\right|^{2}$. To determine the function

$$
\Gamma_{I}\left(\boldsymbol{\rho}_{1}, \boldsymbol{\rho}_{2}, z\right)=\left\langle W_{4}\left(\boldsymbol{\rho}_{1}, \boldsymbol{\rho}_{2}\right)\right\rangle=\left\langle I\left(\boldsymbol{\rho}_{1}, z\right) I\left(\boldsymbol{\rho}_{2}, z\right)\right\rangle,
$$

we will use DWFT solution (74) with respect to the secondorder effects

$$
\begin{aligned}
W_{4}\left(\boldsymbol{\rho}_{1}, \boldsymbol{\rho}_{2}\right) & =E\left(\boldsymbol{\rho}_{1}, z\right) E^{*}\left(\boldsymbol{\rho}_{1}, z\right) E\left(\boldsymbol{\rho}_{2}, z\right) E^{*}\left(\boldsymbol{\rho}_{2}, z\right) \\
& =\left|E_{0}\right|^{4} \widehat{W}_{4}\left(\boldsymbol{\rho}_{1}, \boldsymbol{\rho}_{2}\right),
\end{aligned}
$$

where

$$
\begin{aligned}
& \widehat{W}_{4}\left(\boldsymbol{\rho}_{1}, \boldsymbol{\rho}_{2}\right)=U\left(\boldsymbol{\rho}_{1}, z\right) U^{*}\left(\boldsymbol{\rho}_{1}, z\right) U\left(\boldsymbol{\rho}_{2}, z\right) U^{*}\left(\boldsymbol{\rho}_{2}, z\right) \\
& =\left[\frac{k\left(z-z_{0}\right)}{\pi}\right]^{2} \int_{-\infty}^{\infty} \cdots \int_{-\infty}^{\infty} d^{2} s_{1} \cdots d^{2} p_{4} \\
& \cdot \exp \left\{-i 2 k \bar{\varphi}_{4}+i k \widetilde{\varphi}_{14}+i k \widetilde{\varphi}_{24}(\mathbf{s}, \mathbf{p})\right\} \\
& \bar{\varphi}_{4}=\sum_{m=1}^{4}(-1)^{m+1}\left\{\mathbf{p}_{m} \mathbf{s}_{m}\left(z-z_{0}\right)+\mathbf{s}_{m} \boldsymbol{\rho}_{0}\right\}-\left(\mathbf{p}_{1}-\mathbf{p}_{2}\right) \boldsymbol{\rho}_{1} \\
& +\left(\mathbf{p}_{3}-\mathbf{p}_{4}\right) \boldsymbol{\rho}_{2}, \\
& \widetilde{\varphi}_{14}=0.5 \sum_{m=1}^{4}(-1)^{m+1} \int_{z 0}^{z} \widetilde{\varepsilon}_{1}\left[\overline{\boldsymbol{\rho}}_{m}\left(z^{\prime}\right), z^{\prime}\right] d z^{\prime}, \\
& \widetilde{\varphi}_{24}=0.5 \sum_{m=1}^{4}(-1)^{m+1} \int_{z 0}^{z} \widetilde{\varepsilon}_{2}\left[\overline{\boldsymbol{\rho}}_{m}\left(z^{\prime}\right)+\widetilde{\boldsymbol{\rho}}_{m}\left(z^{\prime}\right), z^{\prime}\right] d z^{\prime}, \\
& \overline{\boldsymbol{\rho}}_{m}\left(z^{\prime}\right)=\mathbf{p}_{m}\left(z^{\prime}-z\right)+\mathbf{s}_{m}\left(z^{\prime}-z_{0}\right), \\
& \widetilde{\boldsymbol{\rho}}_{m}\left(z^{\prime}\right)=\frac{1}{4} \int_{z_{0}}^{z}\left|z^{\prime}-z^{\prime \prime}\right| \frac{\partial}{\partial \boldsymbol{\rho}} \widetilde{\varepsilon}_{1}\left[\overline{\boldsymbol{\rho}}_{m}\left(z^{\prime \prime}\right), z^{\prime}\right] d z^{\prime \prime} .
\end{aligned}
$$

By averaging (95) in small-scale inhomogeneities and taking their small value into account, we get

$$
\begin{aligned}
& \left\langle\widehat{W}_{4}\left(\boldsymbol{\rho}_{1}, \boldsymbol{\rho}_{2}\right) \mid \widetilde{\varepsilon}_{1}\right\rangle=\left[\frac{k\left(z-z_{0}\right)}{\pi}\right]^{2} \\
& \cdot \int_{-\infty}^{\infty} \cdots \int_{-\infty}^{\infty} d^{2} s_{1} \cdots d^{2} p_{4} \\
& \cdot \exp \left\{-i 2 \bar{\varphi}_{4}+i \widetilde{\varphi}_{14}\right\}\left\{1-0.5 D_{24}\right\},
\end{aligned}
$$

where

$$
\begin{aligned}
& D_{24}=k^{2} \pi \int_{z_{0}}^{z} d z_{+} \int_{-\infty}^{\infty} d^{2} \kappa \Phi_{\varepsilon 2}\left(\boldsymbol{\kappa}, z_{+}\right)\{2 \\
& \quad-\exp \left[i \boldsymbol{\kappa} \boldsymbol{\rho}_{12}\left(z_{+}\right)\right]+\exp \left[i \boldsymbol{\kappa} \boldsymbol{\rho}_{13}\left(z_{+}\right)\right] \\
& \quad-\exp \left[i \boldsymbol{\kappa} \boldsymbol{\rho}_{14}\left(z_{+}\right)\right]-\exp \left[i \boldsymbol{\kappa} \boldsymbol{\rho}_{23}\left(z_{+}\right)\right] \\
& \left.\quad+\exp \left[i \boldsymbol{\kappa} \boldsymbol{\rho}_{24}\left(z_{+}\right)\right]-\exp \left[i \boldsymbol{\kappa} \boldsymbol{\rho}_{34}\left(z_{+}\right)\right]\right\}, \\
& \boldsymbol{\rho}_{m n}\left(z_{+}\right)=\overline{\boldsymbol{\rho}}_{m}\left(z_{+}\right)-\overline{\boldsymbol{\rho}}_{n}\left(z_{+}\right)+\widetilde{\boldsymbol{\rho}}_{m}\left(z_{+}\right)-\widetilde{\boldsymbol{\rho}}_{n}\left(z_{+}\right) .
\end{aligned}
$$

As above, in the analysis of the two-frequency mutual coherence function, we take into account relationship (86) of the correlation function with the spectrum and the smoothness of large-scale inhomogeneities (87):

$$
\begin{aligned}
& 0.5 k \int_{z 0}^{z} \widetilde{\varepsilon}_{1}\left[\overline{\boldsymbol{\rho}}_{m}\left(z^{\prime}\right), \eta\right] d \eta \\
& \quad+\frac{\boldsymbol{\kappa}}{4} \int_{z_{0}}^{z}\left|z_{+}-\eta\right| \frac{\partial}{\partial \boldsymbol{\rho}} \widetilde{\varepsilon}_{1}\left[\overline{\boldsymbol{\rho}}_{m}\left(z^{\prime}\right), \eta\right] d \eta \\
& \approx 0.5 k \int_{z 0}^{z} \widetilde{\varepsilon}_{1}\left[\overline{\boldsymbol{\rho}}_{m}\left(z^{\prime}\right)+\frac{\boldsymbol{\kappa}}{2 k}\left|z_{+}-\eta\right|, \eta\right] d \eta .
\end{aligned}
$$

After substituting (99) in (97) and averaging (97) over $\widetilde{\varepsilon}_{1}$ in the resulting integral representation for $\bar{\Gamma}_{I}\left(\rho_{1}, \rho_{2}, z\right)=$ $\left\langle\widehat{W}_{4}\left(\boldsymbol{\rho}_{1}, \boldsymbol{\rho}_{2}\right)\right\rangle$, we turn to new integration variables

$$
\begin{aligned}
& \mathbf{s}_{1,2}=\mathbf{q}_{1}+\frac{\mathbf{v}_{1}}{2} \pm\left(\mathbf{u}_{1}+\frac{\tau_{1}}{2}\right) \\
& \mathbf{s}_{3,4}=\mathbf{q}_{1}-\frac{\mathbf{v}_{1}}{2} \pm\left(\mathbf{u}_{1}-\frac{\tau_{1}}{2}\right) \\
& \mathbf{p}_{1,2}=\mathbf{q}_{2}+\frac{\mathbf{v}_{2}}{2} \pm\left(\mathbf{u}_{2}+\frac{\tau_{2}}{2}\right) \\
& \mathbf{p}_{3,4}=\mathbf{q}_{2}-\frac{\mathbf{v}_{2}}{2} \pm\left(\mathbf{u}_{2}-\frac{\tau_{2}}{2}\right)
\end{aligned}
$$

and evaluate integrals over $\mathbf{q}_{1,2}$ and $\mathbf{u}_{1,2}$. As a result, we obtain

$$
\begin{aligned}
\widehat{\Gamma}_{I}\left(\boldsymbol{\rho}_{1}, \boldsymbol{\rho}_{2}, z\right) & =\widehat{\Gamma}_{I}(\Delta \boldsymbol{\rho}, z) \\
& =\widehat{\Gamma}_{10}+\widehat{\Gamma}_{20}+2 \operatorname{Re}\left[\widehat{\Gamma}_{21}+\widehat{\Gamma}_{22}+\widehat{\Gamma}_{23}\right],
\end{aligned}
$$


where

$$
\begin{aligned}
& \widehat{\Gamma}_{10}(\Delta \boldsymbol{\rho})=\left[\frac{k\left(z-z_{0}\right)}{2 \pi}\right]^{4} \\
& \cdot \int_{-\infty}^{\infty} \int_{-\infty}^{\infty} \int_{-\infty}^{\infty} \int_{-\infty}^{\infty} d^{2} v_{1} d^{2} v_{2} d^{2} \tau_{1} d^{2} \tau_{2} \\
& \cdot \exp \left\{-i 2 k\left[\left(\mathbf{v}_{1} \boldsymbol{\tau}_{2}+\mathbf{v}_{2} \boldsymbol{\tau}_{1}\right)\left(z-\mathrm{z}_{0}\right)-\boldsymbol{\tau}_{2} \Delta \boldsymbol{\rho}\right]\right. \\
& \left.-0.5 \int_{z_{0}}^{z} \widehat{D}_{\varepsilon 1}(\eta, 0,0) d \eta\right\} \text {, } \\
& \widehat{\Gamma}_{20}(\Delta \rho)=-k^{2} \pi\left[\frac{k\left(z-z_{0}\right)}{2 \pi}\right]^{4} \\
& \cdot \int_{-\infty}^{\infty} \int_{-\infty}^{\infty} \int_{-\infty}^{\infty} \int_{-\infty}^{\infty} d^{2} v_{1} d^{2} v_{2} d^{2} \tau_{1} d^{2} \tau_{2} \\
& \cdot \int_{z_{0}}^{z} d z_{+} \int_{-\infty}^{\infty} d^{2} \kappa \Phi_{\varepsilon 2}\left(\kappa, z_{+}\right) \\
& \cdot \exp \left\{-i 2 k\left[\left(\mathbf{v}_{1} \boldsymbol{\tau}_{2}+\mathbf{v}_{2} \boldsymbol{\tau}_{1}\right)\left(z-z_{0}\right)-\boldsymbol{\tau}_{2} \Delta \boldsymbol{\rho}\right]\right. \\
& \left.-0.5 \int_{z_{0}}^{z} \widehat{D}_{\varepsilon 1}[\eta, 0,0] d \eta\right\} \\
& \widehat{\Gamma}_{21}(\Delta \rho)=k^{2} \pi\left[\frac{k\left(z-z_{0}\right)}{2 \pi}\right]^{4} \\
& \cdot \int_{-\infty}^{\infty} \int_{-\infty}^{\infty} \int_{-\infty}^{\infty} \int_{-\infty}^{\infty} d^{2} v_{1} d^{2} v_{2} d^{2} \tau_{1} d^{2} \tau_{2} \\
& \cdot \int_{z_{0}}^{z} d z_{+} \int_{-\infty}^{\infty} d^{2} \kappa \Phi_{\varepsilon 2}\left(\boldsymbol{\kappa}, z_{+}\right) \\
& \cdot \exp \left\{-i 2 k\left[\left(\mathbf{v}_{1} \boldsymbol{\tau}_{2}+\mathbf{v}_{2} \boldsymbol{\tau}_{1}\right)\left(z-z_{0}\right)-\boldsymbol{\tau}_{2} \Delta \boldsymbol{\rho}\right]\right. \\
& +i \boldsymbol{\kappa}\left[\overline{\boldsymbol{\rho}}\left(\boldsymbol{\tau}_{1}, \boldsymbol{\tau}_{2}, z_{+}\right)\right] \\
& \left.-0.5 \int_{z_{0}}^{z} \widehat{D}_{\varepsilon 1}\left[\eta, 0, \frac{-\boldsymbol{\kappa}\left|z_{+}-\eta\right|}{(2 k)}\right] d \eta\right\}, \\
& \widehat{\Gamma}_{22}(\Delta \rho)=k^{2} \pi\left[\frac{k\left(z-z_{0}\right)}{2 \pi}\right]^{4} \\
& \cdot \int_{-\infty}^{\infty} \int_{-\infty}^{\infty} \int_{-\infty}^{\infty} \int_{-\infty}^{\infty} d^{2} v_{1} d^{2} v_{2} d^{2} \tau_{1} d^{2} \tau_{2} \\
& \cdot \int_{z_{0}}^{z} d z_{+} \int_{-\infty}^{\infty} d^{2} \kappa \Phi_{\varepsilon 2}\left(\kappa, z_{+}\right) \\
& \cdot \exp \left\{-i 2 k\left[\left(\mathbf{v}_{1} \boldsymbol{\tau}_{2}+\mathbf{v}_{2} \boldsymbol{\tau}_{1}\right)\left(z-z_{0}\right)-\boldsymbol{\tau}_{2} \Delta \boldsymbol{\rho}\right]\right.
\end{aligned}
$$

$$
\begin{aligned}
\widehat{\Gamma}_{23}(\Delta \boldsymbol{\rho})=k^{2} \pi\left[\frac{k\left(z-z_{0}\right)}{2 \pi}\right]^{4} \\
\cdot \int_{-\infty}^{\infty} \int_{-\infty}^{\infty} \int_{-\infty}^{\infty} \int_{-\infty}^{\infty} d^{2} v_{1} d^{2} v_{2} d^{2} \tau_{1} d^{2} \tau_{2} \\
\cdot \int_{z_{0}}^{z} d z_{+} \int_{-\infty}^{\infty} d^{2} \kappa \Phi_{\varepsilon 2}\left(\boldsymbol{\kappa}, z_{+}\right) \\
\quad \cdot \exp \left\{-i 2 k\left[\left(\mathbf{v}_{1} \boldsymbol{\tau}_{2}+\mathbf{v}_{2} \boldsymbol{\tau}_{1}\right)\left(z-z_{0}\right)-\boldsymbol{\tau}_{2} \Delta \boldsymbol{\rho}\right]\right. \\
+i \boldsymbol{\kappa}\left[\overline{\boldsymbol{\rho}}\left(\mathbf{v}_{1}+\boldsymbol{\tau}_{1}, \mathbf{v}_{2}+\boldsymbol{\tau}_{2}, z_{+}\right)\right] \\
\left.\quad-0.5 \int_{z_{0}}^{z} \widehat{D}_{\varepsilon 1}\left[\eta, \frac{\boldsymbol{\kappa}\left|z_{+}-\eta\right|}{2 k}, \frac{\boldsymbol{\kappa}\left|z_{+}-\eta\right|}{2 k}\right] d \eta\right\} \\
\widehat{D}_{\varepsilon 1}\left[\eta, f_{1}, f_{2}\right]=0.5 k^{2}\left\{2 \bar{\Psi}_{\varepsilon}(0, \eta)\right. \\
\quad-2 \bar{\Psi}_{\varepsilon}\left(\overline{\boldsymbol{\rho}}\left(\boldsymbol{\tau}_{1}, \boldsymbol{\tau}_{2}, \eta\right)+f_{1}, \eta\right)-2 \bar{\Psi}_{\varepsilon}\left(\overline{\boldsymbol{\rho}}\left(\mathbf{v}_{1}, \mathbf{v}_{2}, \eta\right)\right. \\
\left.\quad+f_{2}, \eta\right)+\bar{\Psi}_{\varepsilon}\left(\overline{\boldsymbol{\rho}}\left(\mathbf{v}_{1}+\boldsymbol{\tau}_{1}, \mathbf{v}_{2}+\boldsymbol{\tau}_{2}, \eta\right)+f_{2}+f_{1}, \eta\right) \\
\left.+\bar{\Psi}_{\varepsilon}\left(\overline{\boldsymbol{\rho}}\left(\mathbf{v}_{1}-\boldsymbol{\tau}_{1}, \mathbf{v}_{2}-\boldsymbol{\tau}_{2}, \eta\right)+f_{2}-f_{1}, \eta\right)\right\} \\
\overline{\boldsymbol{\rho}}\left(\mathbf{p}, \mathbf{s}, z^{\prime}\right)=\mathbf{p}\left(z^{\prime}-z\right)+\mathbf{s}\left(z^{\prime}-z_{0}\right) .
\end{aligned}
$$

At small distances, when condition (91) is met and the incident wave distortion effects can be neglected, (101)-(107) yield

$$
\begin{aligned}
\Gamma_{I}(\Delta \boldsymbol{\rho}, z)=\left|E_{0}\right|^{4}\left[\frac{k\left(z-z_{0}\right)}{2 \pi}\right]^{4} \\
\quad \cdot \int_{-\infty}^{\infty} \int_{-\infty}^{\infty} \int_{-\infty}^{\infty} \int_{-\infty}^{\infty} d^{2} v_{1} d^{2} v_{2} d^{2} \tau_{1} d^{2} \tau_{2} \\
\quad \cdot \exp \left\{-i 2 k\left[\left(\mathbf{v}_{1} \boldsymbol{\tau}_{2}+\mathbf{v}_{2} \tau_{1}\right)\left(z-z_{0}\right)-\boldsymbol{\tau}_{2} \Delta \boldsymbol{\rho}\right]\right. \\
\quad-\frac{k^{2}}{4} \int_{z_{0}}^{z}\left\{2 \bar{\Psi}_{\varepsilon}(0, \eta)-2 \bar{\Psi}_{\varepsilon}\left(\boldsymbol{\tau}_{1}\left(\eta-z_{0}\right)\right.\right. \\
\left.+\boldsymbol{\tau}_{2}(\eta-z), \eta\right)-2 \bar{\Psi}_{\varepsilon}\left(\mathbf{v}_{1}\left(\eta-z_{0}\right)\right. \\
\left.\quad+\mathbf{v}_{2}(\eta-z), \eta\right)+\bar{\Psi}_{\varepsilon}\left(\left(\mathbf{v}_{1}+\boldsymbol{\tau}_{1}\right)\left(\eta-z_{0}\right)\right. \\
\left.\quad+\left(\mathbf{v}_{2}+\boldsymbol{\tau}_{2}\right)(\eta-z), \eta\right)+\bar{\Psi}_{\varepsilon}\left(\left(\mathbf{v}_{1}-\boldsymbol{\tau}_{1}\right)\left(\eta-z_{0}\right)\right. \\
\left.\left.+\left(\mathbf{v}_{2}-\boldsymbol{\tau}_{2}\right)(\eta-z)_{1}, \eta\right)\right\} .
\end{aligned}
$$

From expression (109), we can obtain the known results of the phase-screen method, Rytov and geometrical optics approximations for intensity correlation and scintillation index. 
With increasing layer thickness, when (91) is not met, we use the approach of [14] for analyzing (101)-(107). We have to bear in mind that the maximum contribution at great distance belongs to the neighborhoods of the areas $\mathbf{v}_{1}=$ $\mathbf{v}_{2}=0$ and $\boldsymbol{\tau}_{1}=\boldsymbol{\tau}_{2}=0$. Resulting from the asymptotic evaluation of integrals in (102)-(106) with $\left|z-z_{0}\right| \rightarrow \infty$ for normalized correlation function $K_{I}(\Delta \rho)=\Psi_{I}(\Delta \rho) /\langle I\rangle^{2}=$ $\Gamma_{I}(\Delta \rho, z) /\langle I\rangle^{2}-1$, we get

$$
K_{I}(\Delta \boldsymbol{\rho}) \approx B_{I}^{(1)}(\Delta \boldsymbol{\rho})+B_{I}^{(2)}(\Delta \boldsymbol{\rho})+B_{I}^{(2)}(\Delta \boldsymbol{\rho}),
$$

where

$$
\begin{aligned}
& B_{I}^{(1)}(\Delta \boldsymbol{\rho})=\exp \left\{-0.5 k^{2} \int_{z_{0}}^{z}\left[\bar{\Psi}_{\varepsilon}(0, \eta)-\bar{\Psi}_{\varepsilon}\left(\frac{\Delta \boldsymbol{\rho}\left(\eta-z_{0}\right)}{\left(z-z_{0}\right)}, \eta\right)\right] d \eta\right\} \\
& B_{I}^{(2)}(\Delta \boldsymbol{\rho})=k^{2} \pi \int_{z_{0}}^{z} d z_{+} \int_{-\infty}^{\infty} d^{2} \kappa \Phi_{\varepsilon_{2}}\left(\boldsymbol{\kappa}, 0, z_{+}\right)\left\{1-\cos \left[\frac{\kappa^{2}\left(z_{+}-z\right)\left(z_{+}-z_{0}\right)}{k\left(z-z_{0}\right)}\right]\right\} \cos \left[\frac{\Delta \boldsymbol{\rho} \boldsymbol{\kappa}\left(z_{+}-z_{0}\right)}{\left(z-z_{0}\right)}\right] \exp \left\{\frac{-k^{2}}{2}\right. \\
& \left.\quad \cdot \int_{z_{0}}^{z}\left[\bar{\Psi}_{\varepsilon 1}(0, \eta)-\bar{\Psi}_{\varepsilon 1}\left(\frac{\boldsymbol{\kappa} \boldsymbol{\rho}_{c}(\eta)}{k}, \eta\right)\right] d \eta\right\}, \\
& B_{I}^{(3)}(\Delta \boldsymbol{\rho})=k^{2} \pi \int_{z_{0}}^{z} d z_{+} \int_{-\infty}^{\infty} d^{2} \kappa \Phi_{\varepsilon_{2}}\left(\boldsymbol{\kappa}, 0, z_{+}\right)\left\{1-\cos \left[\kappa^{2} \frac{\left(z_{+}-z\right)\left(z_{+}-z_{0}\right)}{k\left(z-z_{0}\right)}+\boldsymbol{\kappa} \Delta \boldsymbol{\rho} \frac{z_{+}-z_{0}}{z-z_{0}}\right]\right\} \\
& \cdot \exp \left\{\frac{-k^{2}}{2} \int_{z_{0}}^{z}\left[\bar{\Psi}_{\varepsilon 1}(0, \eta)-\bar{\Psi}_{\varepsilon 1}\left(\frac{\boldsymbol{\kappa} \boldsymbol{\rho}_{c}(\eta)}{k}+\Delta \boldsymbol{\rho} \frac{\eta-z_{0}}{z-z_{0}}, \eta\right)\right] d \eta\right\} \\
& \boldsymbol{\rho}_{c}(\eta)=\frac{1}{z-z_{0}}\left\{\begin{array}{l}
\left(z_{+}-z\right)\left(\eta-z_{0}\right) \\
\left(z_{+}-z_{0}\right)(\eta-z) \quad \eta>z_{+}
\end{array}\right.
\end{aligned}
$$

Hence, for the scintillation index $\beta^{2}$ of a wave propagating in a multiscale randomly inhomogeneous medium, we get

$$
\begin{aligned}
\beta^{2} & =K_{I}(0)=1+2 k^{2} \pi \int_{z_{0}}^{z} d z_{+} \int_{-\infty}^{\infty} d^{2} \kappa \Phi_{\varepsilon_{2}}\left(\boldsymbol{\kappa}, 0, z_{+}\right) \\
& \cdot\left\{1-\cos \left[\frac{\kappa^{2}\left(z_{+}-z\right)\left(z_{+}-z_{0}\right)}{k\left(z-z_{0}\right)}\right]\right\} \exp \left\{\frac{-k^{2}}{2}\right. \\
& \left.\cdot \int_{z_{0}}^{z}\left[\bar{\Psi}_{\varepsilon 1}(0, \eta)-\bar{\Psi}_{\varepsilon 1}\left(\frac{\boldsymbol{\kappa} \boldsymbol{\rho}_{c}(\eta)}{k}, \eta\right)\right] d \eta\right\} .
\end{aligned}
$$

With respect to the filtering effect of the coefficient $\{1-$ $\left.\cos \left[\kappa^{2}\left(z_{+}-z\right)\left(z_{+}-z_{0}\right) /\left(k\left(z-z_{0}\right)\right)\right]\right\}$ in (112), (113), and (115), we can substitute the spectrum of small-scale inhomogeneities $\Phi_{\varepsilon_{2}}\left(\boldsymbol{\kappa}, 0, z_{+}\right)$with the full spectrum $\Phi_{\varepsilon}\left(\boldsymbol{\kappa}, 0, z_{+}\right)=$ $\Phi_{\varepsilon_{1}}\left(\boldsymbol{\kappa}, 0, z_{+}\right)+\Phi_{\varepsilon_{2}}\left(\boldsymbol{\kappa}, 0, z_{+}\right)$. Since the contribution of smallscale inhomogeneities is small, we can substitute $\bar{\Psi}_{\varepsilon 1}(0, \eta) \approx$ $\bar{\Psi}_{\varepsilon}(0, \eta)=\bar{\Psi}_{\varepsilon 1}(0, \eta)+\bar{\Psi}_{\varepsilon 2}(0, \eta)$ in (112), (113), and (115). As a result, we get expressions for the correlation function $K_{I}(\Delta \rho)$ and scintillation index $\beta^{2}$, for a plane wave such that $z_{0} \rightarrow-\infty$ go to results [14] of asymptotic computation of the strong expressions obtained by the path integral method.

\section{Conclusion}

The use of the DWFT method and hybrid approach together enabled us to derive expressions for statistical characteristics of the wave field in a multiscale randomly inhomogeneous medium. Upon that we took into account the second-order effects associated with the ray trajectory variations within sizes of small-scale inhomogeneities. It is shown that, similar to the Markov approximation, the mean field and the spatial coherence function coincide with their phase geometrical optics approximations. Within the DWFT, we were able to find a solution for the frequency coherence function and to show that, for narrow-band signals, the second-order effects do not significantly affect the frequency correlation. Moreover, it is shown that, with decreasing bandwidth, phase geometrical optics approximation can be used to calculate frequency correlation.

A more significant role in the intensity correlation belongs to the second-order effects. Analysis of the obtained expressions showed that while the second-order effects can be neglected for the small thickness of the inhomogeneous layer, these effects become noticeable as the layer thickness increases. It is interesting to note that the resulting intensity correlation function at large distances goes to the results obtained earlier by the path integral method. This shows that the intensity fluctuations of a wave propagating in a multiscale randomly inhomogeneous medium at great distance are significantly affected by the second-order effects, that is, the incident wave distortion effects.

\section{Conflicts of Interest}

The author declares that there are no conflicts of interest regarding the publication of this paper. 


\section{Acknowledgments}

The author is very grateful to S. V. Filippova for her assistance in preparing the English version of the manuscript. This work was supported by a project part of the Government Assignment for Scientific Research from the Ministry of Education and Science, Russia (no. 3.903.2017/4.6).

\section{References}

[1] Y. A. Kravtsov and Y. I. Orlov, Geometrical Optics of Inhomogeneous Media, Springer, Berlin, Germany, 1990.

[2] S. M. Rytov, Y. A. Kravtsov, and V. I. Tatarskii, Introduction to Statistical Radiophysics. Vol. 4, Wave Propagation Through Random Media, Springer, New York, NY, USA, 1989.

[3] A. Ishimaru, Wave Propagation and Scattering in Random Media. Vol 2. Multiple Scattering, Turbulence, Rough Surfaces and Remote Sensing, Academic Press, New York, NY, USA, 1978.

[4] V. I. Tatarskii, "The Effect of a Turbulent Atmosphere on Wave Propagation,” Tech. Rep., National Technical Information Service, Springfield, Va, USA, 1971.

[5] Y. A. Kravtsov, S. I. Feyzulin, and A. G. Vinogradov, Radio Wave Propagation through The Earth's Atmosphere, M.: Radio i Svyaz, 1983.

[6] V. E. Kunitsyn and E. D. Tereshchenko, Ionospheric Tomography, Springer, Berlin, Germany, 2003.

[7] Y. N. Barabanenkov, Y. A. Kravtsov, S. M. Rytov, and V. I. Tamarskilu, "Status of the theory of propagation of waves in a randomly inhomogeneous medium," Soviet Physics Uspekhi, vol. 13, no. 5, pp. 551-575, 1971.

[8] B. N. Gershman, L. M. Erukhimov, and Y. Y. Yashin, Wave Phenomena in The Ionosphere and Space Plasma, M.: FIZMATLIT, 1984.

[9] Y. A. Kravtsov, "Propagation of electromagnetic waves through a turbulent atmosphere," Reports on Progress in Physics, vol. 55, no. 1, article 002, pp. 39-112, 1992.

[10] E. N. Bramley, "The diffraction of waves by an irregular refracting medium," Proceedings of the Royal Society A Mathematical, Physical and Engineering Sciences, vol. 225, pp. 515-518, 1954.

[11] L. M. Erukhimov and V. P. Uryadov, "Frequency correlation of radio-wave fluctuations behind n random screens," Radiophysics and Quantum Electronics, vol. 11, no. 12, pp. 1050-1058, 1972.

[12] V. E. Gherm, N. N. Zernov, and H. J. Strangeways, "Propagation model for transionospheric fluctuating paths of propagation: simulator of the transionospheric channel," Radio Science, vol. 40, no. 1, pp. RS1003-RS10003, 2005.

[13] R. L. Fante, “Two-position, two-frequency mutual-coherence function in turbulence," Journal of the Optical Society of America, vol. 71, no. 12, pp. 1446-1451, 1981.

[14] V. I. Klyatskin, Stochastic Equations and Waves in Random Inhomogeneous Media, 1980, [Translation: Ondes et Equations Stochastique dans les Milieux Aleatoirement Non-homogenes, Editions de Physiques, Besancon, 1985 (In French)].

[15] M. I. Charnotskii, J. Gozani, V. I. Tatarskii, and V. U. Zavorotny, "IV wave propagation theories in random media based on the path-integral approach," Progress in Optics, vol. 32, pp. 203-266, 1993.

[16] V. P. Kandidov, "Monte Carlo method in nonlinear statistical optics," Physics-Uspekhi, vol. 39, no. 12, pp. 1243-1272, 1996.
[17] D. L. Knepp, "Multiple phase-screen calculation of the temporal behavior of stochastic waves," Proceedings of the IEEE, vol. 71, no. 6, pp. 722-737, 1983.

[18] J. M. Martin and S. M. Flatté, "Intensity images and statistics from numerical simulation of wave propagation in 3-D random media," Applied Optics, vol. 27, no. 11, pp. 2111-2126, 1988.

[19] C. H. Liu and K. C. Yeh, "Frequency and spatial correlation functions in a fading communication channel through the ionosphere," Radio Science, vol. 10, no. 12, pp. 1055-1061, 1975.

[20] A. A. Bitjukov, V. E. Gherm, and N. N. Zernov, "On the solution of Markov's parabolic equation for the second-order spaced frequency and position coherence function," Radio Science, vol. 37, no. 4, pp. 19-1-19-9, 2002.

[21] Y. A. Kravtsov and Y. I. Orlov, Caustics, Catastrophes and Wave Fields, Springer, Berlin, Germany, 1999.

[22] M. V. Tinin, "Quasi-geometrical optics methods in the theory of wave propagation through a randomly inhomogeneous layer," Waves in Random and Complex Media, vol. 8, no. 3, pp. 329338, 1998.

[23] R. Mazar, "High-frequency propagators for diffraction and backscattering in random media," Journal of the Optical Society of America A: Optics and Image Science, and Vision, vol. 7, no. 1, pp. 34-46, 1990.

[24] S. Frankenthal, "The mutual coherence function in a scattering channel-a two scale solution," The Journal of the Acoustical Society of America, vol. 85, no. 1, pp. 104-113, 1989.

[25] G. Samelsohn, "High-frequency directed wave propagators: a path integral derivation," IEEE Transactions on Antennas and Propagation, vol. 61, no. 11, pp. 5637-5648, 2013.

[26] Y. A. Kravtsov and M. V. Tinin, "Representation of a wave field in a randomly inhomogeneous medium in the form of the double-weighted Fourier transform," Radio Science, vol. 35, no. 6, pp. 1315-1322, 2000.

[27] M. V. Tinin, S. N. Kolesnik, and Y. A. Kravtsov, "An integral representation for the field of the wave propagating in a medium with random inhomogeneities of different scales," Proceedings of SPIE - The International Society for Optical Engineering, vol. 4678, pp. 605-609, 2002.

[28] Y. A. Kravtsov and M. V. Tinin, "Ray based diffraction tomography of the ionosphere and laboratory inhomogeneous plasma," Cosmic Research, vol. 41, no. 4, pp. 357-358, 2003.

[29] Y. A. Kravtsov, M. V. Tinin, and S. I. Knizhnin, "Diffraction tomography of inhomogeneous medium in the presence of strong phase variations," Journal of Communications Technology and Electronics, vol. 56, no. 7, pp. 831-837, 2011.

[30] M. V. Tinin and S. I. Knizhin, "Eliminating the effects of multipath signal propagation in a smoothly inhomogeneous medium," Radiophysics and Quantum Electronics, vol. 56, no. 7, pp. 413-421, 2013.

[31] B. C. Kim and M. V. Tinin, "Contribution of ionospheric irregularities to the error of dual-frequency GNSS positioning," Journal of Geodesy, vol. 81, no. 3, pp. 189-199, 2007. 


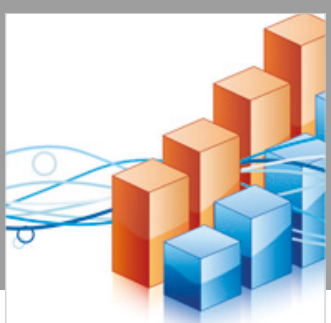

Advances in

Operations Research

\section{-n-m}
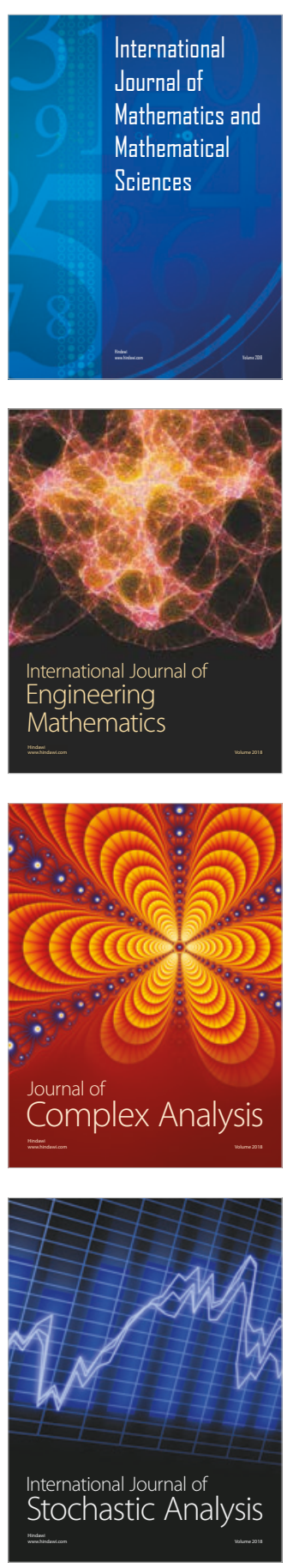
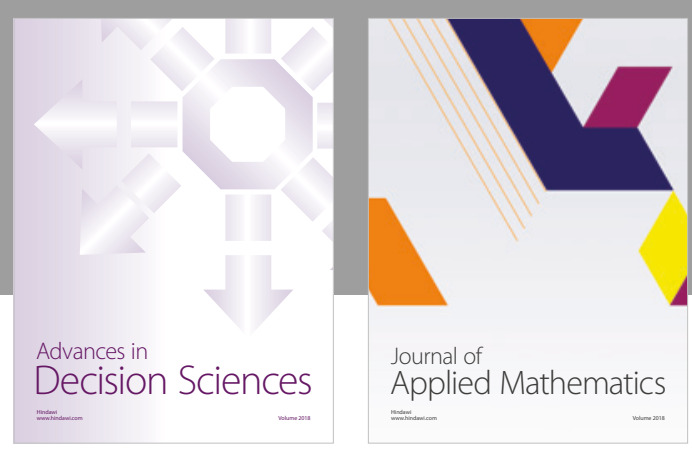

Journal of

Applied Mathematics
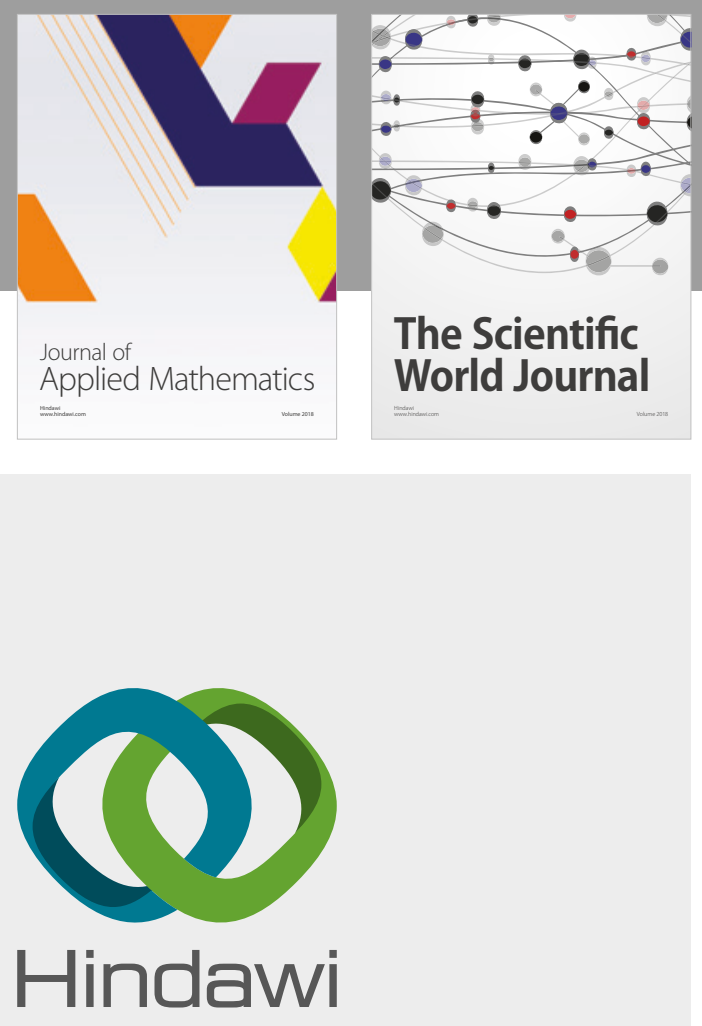

Submit your manuscripts at

www.hindawi.com

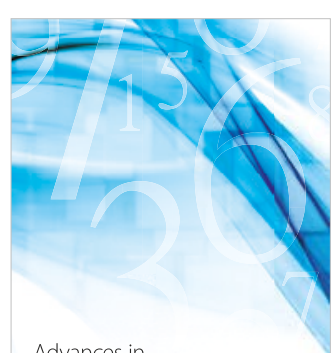

Advances in
Numerical Analysis
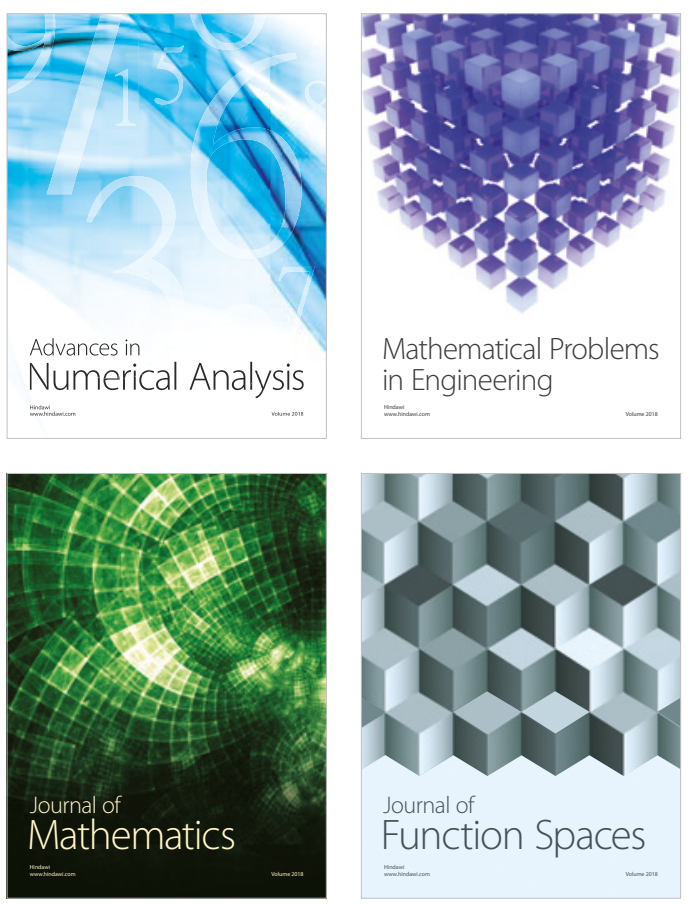

Mathematical Problems in Engineering

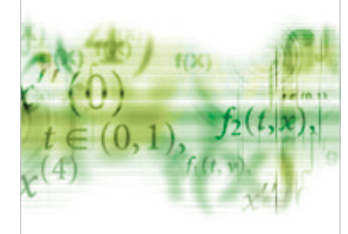

International Journal of

Differential Equations

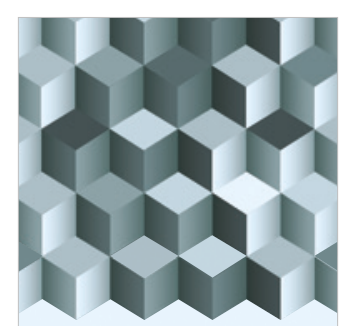

Journal of

Function Spaces

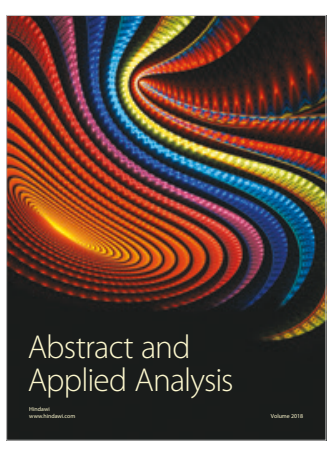

The Scientific

World Journal

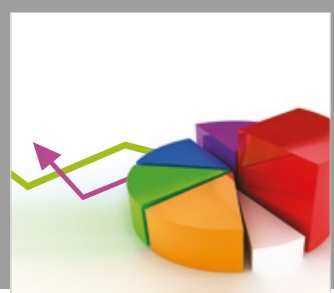

Journal of

Probability and Statistics
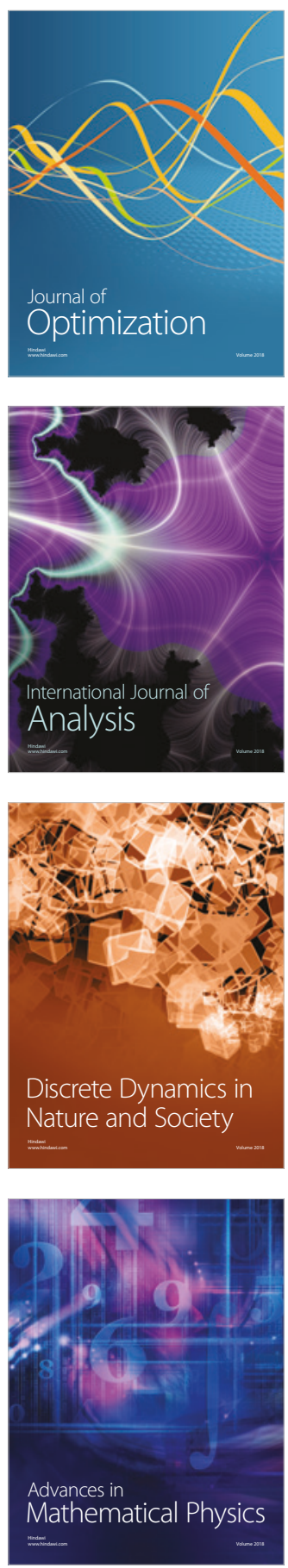